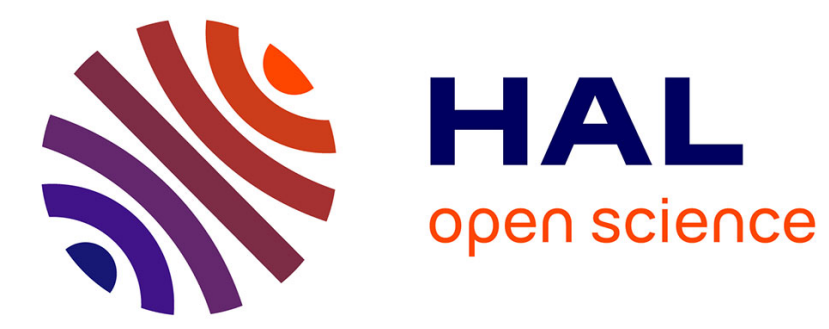

\title{
OPTIMAL CONTROL OF A POPULATION DYNAMICS MODEL WITH MISSING BIRTH RATE
}

\author{
Cyrille Kenne, Günter Leugering, Gisèle Mophou
}

\section{To cite this version:}

Cyrille Kenne, Günter Leugering, Gisèle Mophou. OPTIMAL CONTROL OF A POPULATION DYNAMICS MODEL WITH MISSING BIRTH RATE. SIAM Journal on Control and Optimization, In press. hal-02093551

\section{HAL Id: hal-02093551 https://hal.science/hal-02093551}

Submitted on 11 Aug 2020

HAL is a multi-disciplinary open access archive for the deposit and dissemination of scientific research documents, whether they are published or not. The documents may come from teaching and research institutions in France or abroad, or from public or private research centers.
L'archive ouverte pluridisciplinaire HAL, est destinée au dépôt et à la diffusion de documents scientifiques de niveau recherche, publiés ou non, émanant des établissements d'enseignement et de recherche français ou étrangers, des laboratoires publics ou privés. 


\title{
OPTIMAL CONTROL OF A POPULATION DYNAMICS MODEL WITH MISSING BIRTH RATE*
}

\author{
CYRILLE KENNE ${ }^{\dagger}$, GÜNTER LEUGERING ${ }^{\ddagger}$, AND GISÈLE MOPHOU $§$
}

\begin{abstract}
We consider a model of population dynamics with age dependence and spatial structure but unknown birth rate. Using the notion of Low-regret [1], we prove that we can bring the state of the system to a desired state by acting on the system via a localized distributed control. We provide the optimality systems that characterize the Low-regret control. Moreover, using an appropriate Hilbert space, we prove that the family of Low-regret controls tends to a so-called Noregret control, which we, in turn, characterize.
\end{abstract}

Key words. Population dynamics, incomplete data, optimal control, No-regret control, Lowregret control, Euler-Lagrange formula.

AMS subject classifications. 49J20, 92D25, 93C41

1. Introduction. In the modeling of the dynamics of some invasive species governed by diffusive systems with age dependency(for instance invasive plants in bounded domains), it may not be possible to have sufficient observations to obtain a good approximation of the birth function or/and the mortality function [2]. In order to avoid the disappearance of the other species it seems natural to control those invasive species. More precisely, let $\Omega \subset \mathbb{R}^{3}$ be a bounded domain in which the invasive species live. We denote by $\Gamma$ the boundary of the domain and we assume that it is of class $C^{2}$. For the time $T>0$ and the life expectancy of an individual $A>0$, we set $U=(0, T) \times(0, A), Q=U \times \Omega, \Sigma=U \times \Gamma, Q_{A}=(0, A) \times \Omega, Q_{T}=(0, T) \times \Omega$ and $Q_{\omega}=U \times \omega$, where $\omega$ is a non-empty open subset of $\Omega$. Then we consider a model describing the dynamics of an invasive species with age dependence and spatial structure:

$$
\left\{\begin{aligned}
\frac{\partial y}{\partial t}+\frac{\partial y}{\partial a}-\Delta y+\mu y & =f+v \chi_{\omega} & & \text { in } Q, \\
y & =0 & & \text { on } \Sigma, \\
y(0, \cdot, \cdot) & =y^{0} & & \text { in } Q_{A}, \\
y(\cdot, 0, \cdot) & =\int_{0}^{A} g(a) y(t, a, x) d a & & \text { in } Q_{T},
\end{aligned}\right.
$$

where

- $y=y(t, a, x)$ is the distribution of individuals of age $a \geq 0$, at time $t \geq 0$ and location $x \in \Omega$.

- The recruitment $f \in L^{2}(Q)$ is a positive periodic function.

- The control $v \in L^{2}(Q)$ which corresponds to the removal of the individuals in a sub-domain $\omega$ of $\Omega$ and $\chi_{\omega}$ denote the characteristic function of the control set $\omega$.

\footnotetext{
* Submitted to the editors DATE.

Funding: This work was funded by the Fog Research Institute under contract no. FRI- 454 .

${ }^{\dagger}$ University of Buea, Department of Mathematics, Buea, Cameroon (kenne853@gmail.com).

${ }_{\ddagger}^{\ddagger}$ Department Mathematik, Friedrich-Alexander-Universität Erlangen-Nürnberg, Cauerstr. 11 (03.322), 91058 Erlangen, Germany (guenter.leugering@fau.de).

$\S$ African Institute for Mathematical Sciences (AIMS), P.O. Box 608, Limbe Crystal Gardens, South West Region, Cameroon-Laboratoire LAMIA, Université des Antilles, Campus Fouillole, 97159 Pointe-à-Pitre Guadeloupe (FWI)- Laboratoire MAINEGE, Université Ouaga 3S, 06 BP 10347 Ouagadougou 06, Burkina Faso (gisele.mophou@univ-antilles.fr).
} 
- The mortality rate $\mu=\mu(a) \geq 0$ is a known increasing positive function which is continuous on $[0, A]$, whereas the fertility rate $g=g(a) \in L^{\infty}(0, A)$ is unknown and positive.

We assume as in [3], that:

$$
\left(H_{1}\right) \lim _{a \rightarrow A} \int_{0}^{a} \mu(s) d s=+\infty,
$$

which means that each individual in the population dies before age $A$. For more literature on the population dynamics model and the signification of assumption $\left(H_{1}\right)$, we refer to $[3,4,5,6,7,8,9]$ and the reference therein.

Remark 1.1. Set

$$
W(T, A)=\left\{\rho \in L^{2}\left(U ; H_{0}^{1}(\Omega)\right) ; \frac{\partial \rho}{\partial t}+\frac{\partial \rho}{\partial a} \in L^{2}\left(U ; H^{-1}(\Omega)\right)\right\} .
$$

Then we have (see [10]) that

$$
W(T, A) \subset \mathcal{C}\left([0, T], L^{2}\left(Q_{A}\right)\right) \text { and } W(T, A) \subset \mathcal{C}\left([0, A], L^{2}\left(Q_{T}\right)\right) .
$$

Under the assumption on the data, (1.1) has a solution $y(v, g)=y(t, a, x ; v, g)$ in $W(T, A)$. We define the cost function

$$
J(v, g)=\left\|y(v, g)-z_{d}\right\|_{L^{2}(Q)}^{2}+N\|v\|_{L^{2}\left(Q_{\omega}\right)}^{2},
$$

where $z_{d} \in L^{2}(Q)$ and $N>0$ are given.

In this paper, we don't want to determine the unknown fertility rate $g$. Actually, we want to bring the distribution of individuals to a desired distribution $z_{d}$ by acting on the system via a control $v$. In other words, we are interested in solving the following optimization problem:

$$
\inf _{v \in L^{2}\left(Q_{\omega}\right)} \sup _{g \in L^{2}(0, A)} J(v, g) .
$$

But observing that we could have $\sup _{g \in L^{2}(0, A)} J(v, g)=+\infty$, we consider the optimization problem:

$$
\inf _{v \in L^{2}\left(Q_{\omega}\right)} \sup _{g \in L^{2}(0, A)}(J(v, g)-J(0, g)) .
$$

Problem (1.5) is called the No-regret control problem. The notions of No-regret control and Low-regret control were introduced by J. -L. Lions [1] in order to control a phenomenon described by a parabolic equation with missing initial condition. Let us recall that one obtains the Low-regret control problem by relaxing the No-regret control one. See (3.23),(3.24),(3.25) for the relaxation used in this article. By means of the Legendre-Fenchel transform, we prove that the Low-regret control problem is equivalent to a classical optimal control problem. The most difficult task is to prove that this family of controls (called Low-regret controls) converges towards the No-regret control. Also in [11], J. -L. Lions proved that these notion can be used in the framework of decomposition methods. In [12], O. Nakoulima et al. applied this notion to linear evolution equations with incomplete data and they proved that the Low-regret controls converges to the No-regret control for which they obtained a singular optimality system. B. Jacob et al. [13] generalized the notion of No-regret control to population dynamics with incomplete initial data with a distributed control 
acting on the whole domain. They proved the existence and uniqueness of the Noregret control and gave a singular optimality system that characterizes this control. In the nonlinear case, this notion was considered by O. Nakoulima et al. [14] to control on the whole domain a nonlinear system with incomplete data. Observing on the one hand that the No-regret control is typically not easy to characterize and, on the other hand that the Low-regret cost function may not be convex, they proved by adapting this cost to a No-regret control that the adapted Low-regret control converge towards this No-regret control characterized by a singular optimality system. In [15], J. Vélin studied systems governed by quasilinear equations with unknown boundary condition and a control acting on the whole domain. After established some regularity results for the control-to-state and control-perturbation applications and its derivatives, he proved by proceeding as in [14] that the adapted Low-regret control converge towards a No-regret control characterized gain by a singular optimality. Note that in the above papers, the convergence of the Low-regret control towards the No-regret control is obtained by controlling on the whole domain.

In this paper, we use the notion of No-regret and Low-regret to control a model describing the dynamics of population with age dependence and spatial structure with missing birth rate by acting on a part of the domain. Observing that with an unknown the birth rate, the control problem considered is now non-linear, we start by proving some regularity results. Then we prove the existence of a No-regret control. We then regularize the No-regret control problem to a Low-regret control problem $((3.23),(3.24),(3.25))$. We introduce an appropriate Hilbert space to obtain estimates on the states satisfying the optimality systems and by that characterize the Low-regret control. Then we prove that the adapted Low-regret control converges towards a Noregret control and establish a singular optimality system that, in turn, characterizes this no-regret control.

The rest of this paper is structured as follows. In section 2, we give some regularity results. We study the Low-regret and no-regret control and their characterizations in section 3. A conclusion is given in section 4 .

2. Preliminary results. In order to solve the optimization problem (3.2), we need some preliminary results.

In what follows, we adopt the following notation

$$
\left\{\begin{aligned}
L & =\frac{\partial}{\partial t}+\frac{\partial}{\partial a}-\Delta+\mu I, \\
L^{*} & =-\frac{\partial}{\partial t}-\frac{\partial}{\partial a}-\Delta+\mu I,
\end{aligned}\right.
$$

where $I$ is the identity operator.

Proposition 2.1. Let $y=y(v, g)$ be a solution of (1.1). Then the application $(v, g) \mapsto y(v, g)$ is a continuous function from $L^{2}\left(Q_{\omega}\right) \times L^{2}(0, A)$ onto $L^{2}\left(U, H_{0}^{1}(\Omega)\right)$.

Proof. Let $\left(v_{0}, g_{0}\right) \in L^{2}\left(Q_{\omega}\right) \times L^{2}(0, A)$. We show that $(v, g) \mapsto y(v, g)$ is continuous at $\left(v_{0}, g_{0}\right)$.

Set $\bar{y}=y(v, g)-y\left(v_{0}, g_{0}\right)$, then $\bar{y}$ is solution to the problem

$$
\left\{\begin{aligned}
L \bar{y} & =v \chi_{Q_{\omega}}-v_{0} \chi_{Q_{\omega}} & & \text { in } Q \\
\bar{y} & =0 & & \text { on } \Sigma, \\
\bar{y}(0, \cdot, \cdot) & =0 & & \text { in } Q_{A} \\
\bar{y}(\cdot, 0, \cdot) & =\eta & & \text { in } Q_{T}
\end{aligned}\right.
$$


where for $(t, x) \in Q_{T}$,

$$
\eta(t, x)=\int_{0}^{A}\left[g(a) y(t, a, x ; v, g)-g_{0}(a) y\left(t, a, x ; v_{0}, g_{0}\right)\right] d a .
$$

If we set $z=e^{-r t} \bar{y}$ with $r>0$, then we obtain that $z$ is solution to the problem

$$
\left\{\begin{aligned}
L z+r z & =e^{-r t}\left(v \chi_{Q_{\omega}}-v_{0} \chi_{Q_{\omega}}\right) & & \text { in } Q, \\
z & =0 & & \text { on } \Sigma, \\
z(0, \cdot, \cdot) & =0 & & \text { in } Q_{A}, \\
z(\cdot, 0, \cdot) & =e^{-r t} \eta & & \text { in } Q_{T} .
\end{aligned}\right.
$$

Multiplying the first equation of system (2.3) by $z$ and integrating by parts over $Q$, we get

$$
\begin{aligned}
\int_{Q_{\omega}} e^{-r t}\left(v-v_{0}\right) z d x d t d a & =\frac{1}{2}\|z(T, \cdot, \cdot)\|_{L^{2}\left(Q_{A}\right)}^{2}-\frac{1}{2}\|z(0, \cdot, \cdot)\|_{L^{2}\left(Q_{A}\right)}^{2} \\
& +\frac{1}{2}\|z(\cdot, A, \cdot)\|_{L^{2}\left(Q_{T}\right)}^{2}-\frac{1}{2}\|z(\cdot, 0, \cdot)\|_{L^{2}\left(Q_{T}\right)}^{2} \\
& +\|\nabla z\|_{L^{2}(Q)}^{2}+\int_{Q}(r+\mu) z^{2}
\end{aligned}
$$

From this we deduce that

$$
\|\nabla z\|_{L^{2}(Q)}^{2}+r\|z\|_{L^{2}(Q)}^{2} \leq \frac{1}{2}\|z(\cdot, 0, \cdot)\|_{L^{2}\left(Q_{T}\right)}^{2}+\frac{1}{2}\left\|v-v_{0}\right\|_{L^{2}\left(Q_{\omega}\right)}^{2}+\frac{1}{2}\|z\|_{L^{2}(Q)}^{2}
$$

because $\mu \geq 0$. On the other hand, observing that for $(t, x) \in Q_{T}$,

$$
\begin{aligned}
z(t, 0, x) & =e^{-r t} \int_{0}^{A}\left[g(a) y(t, a, x, v, g)-g_{0}(a) y\left(t, a, x, v_{0}, g_{0}\right)\right] d a \\
& =e^{-r t} \int_{0}^{A}\left[\left(g(a)-g_{0}(a)\right) y(t, a, x, v, g)\right] d a+\int_{0}^{A} g_{0}(a) z d a,
\end{aligned}
$$

we obtain

$$
\|z(\cdot, 0, \cdot)\|_{L^{2}\left(Q_{T}\right)}^{2} \leq 2\left\|g-g_{0}\right\|_{L^{2}(0, A)}^{2}\|y\|_{L^{2}(Q)}^{2}+2\left\|g_{0}\right\|_{L^{2}(0, A)}^{2}\|z\|_{L^{2}(Q)}^{2} .
$$

Thus, (2.4) gives

$$
\begin{aligned}
\|\nabla z\|_{L^{2}(Q)}^{2}+\left(r-\left\|g_{0}\right\|_{L^{2}(0, A)}^{2}-\frac{1}{2}\right)\|z\|_{L^{2}(Q)}^{2} & \leq\left\|g-g_{0}\right\|_{L^{2}(0, A)}^{2}\|y\|_{L^{2}(Q)}^{2} \\
& +\frac{1}{2}\left\|v-v_{0}\right\|_{L^{2}\left(Q_{\omega}\right)}^{2} .
\end{aligned}
$$

Choosing $r$ such that $r>\left\|g_{0}\right\|_{L^{2}(0, A)}^{2}+\frac{1}{2}$, we have

$$
\|z\|_{L^{2}\left(U ; H_{0}^{1}(\Omega)\right)}^{2} \leq\left\|g-g_{0}\right\|_{L^{2}(0, A)}^{2}\|y\|_{L^{2}(Q)}^{2}+\frac{1}{2}\left\|v-v_{0}\right\|_{L^{2}\left(Q_{\omega}\right)}^{2} .
$$

From this we deduce

$$
\|z\|_{L^{2}\left(U ; H_{0}^{1}(\Omega)\right)} \leq\left\|g-g_{0}\right\|_{L^{2}(0, A)}\|y\|_{L^{2}(Q)}+\frac{\sqrt{2}}{2}\left\|v-v_{0}\right\|_{L^{2}\left(Q_{\omega}\right)} .
$$


Therefore,

$$
\|\bar{y}\|_{L^{2}\left(U ; H_{0}^{1}(\Omega)\right)} \leq e^{r T}\left\|g-g_{0}\right\|_{L^{2}(0, A)}\|y\|_{L^{2}(Q)}+\frac{\sqrt{2}}{2} e^{r T}\left\|v-v_{0}\right\|_{L^{2}\left(Q_{\omega}\right)} .
$$

As $(v, g) \rightarrow\left(v_{0}, g_{0}\right)$, we have $\bar{y} \rightarrow 0$ strongly in $L^{2}\left(U ; H_{0}^{1}(\Omega)\right)$. Hence $y(v, g) \rightarrow$ $y\left(v_{0}, g_{0}\right)$ strongly in $L^{2}\left(U ; H_{0}^{1}(\Omega)\right)$ as $(v, g) \rightarrow\left(v_{0}, g_{0}\right)$.

Proposition 2.2. Let $\lambda>0$. Let $g, h \in L^{2}(0, A)$ and $v, w \in L^{2}\left(Q_{\omega}\right)$. Let also $y=y(v, g)$ be a solution of (1.1). Set $\bar{y}_{\lambda}=\frac{y(v+\lambda w, g+\lambda h)-y(v, g)}{\lambda}$. Then $\left(\bar{y}_{\lambda}\right)$ converges strongly in $L^{2}\left(U ; H_{0}^{1}(\Omega)\right)$ as $\lambda \rightarrow 0$ to a function $\bar{y}$ which is solution of

$$
\left\{\begin{array}{rlrl}
L \bar{y} & =w \chi_{Q_{\omega}} & & \text { in } Q, \\
\bar{y} & =0 & & \text { on } \Sigma, \\
\bar{y}(0, \cdot, \cdot) & =0 & \text { in } Q_{A}, \\
\bar{y}(\cdot, 0, \cdot) & =\int_{0}^{A} g(a) \bar{y} d a+\int_{0}^{A} h(a) y(t, a, x ; v, g) d a & \text { in } Q_{T} .
\end{array}\right.
$$

Proof. $\bar{y}_{\lambda}$ is a solution to the problem

$28\left\{\begin{aligned} L \bar{y}_{\lambda} & =w \chi_{Q_{\omega}} & \text { in } Q, \\ \bar{y}_{\lambda} & =0 & \text { on } \Sigma, \\ \left.\bar{y}_{\lambda}(0, \cdot, \cdot) \cdot\right) & =0 & \text { in } Q_{A}, \\ \bar{y}_{\lambda}(\cdot, 0, \cdot) & =\int_{0}^{A} g(a) \bar{y}_{\lambda} d a+\int_{0}^{A} h(a) y(t, a, x ; v+\lambda w, g+\lambda h) d a & \text { in } Q_{T} .\end{aligned}\right.$

where for $(t, x) \in Q_{T}$,

$$
\eta_{1}(t, x)=\int_{0}^{A} h(a)[y(t, a, x ; v+\lambda w, g+\lambda h)-y(t, a, x ; v, g)] d a .
$$

We set $z_{\lambda}=e^{-r t} y_{\lambda}$ with $r>0$. Then we obtain that $z_{\lambda}$ is a solution to the problem

$$
\left\{\begin{aligned}
L z_{\lambda}+r z_{\lambda} & =0 & & \text { in } Q, \\
z_{\lambda} & =0 & & \text { on } \Sigma, \\
z_{\lambda}(0, \cdot, \cdot) & =0 & & \text { in } Q_{A}, \\
z_{\lambda}(\cdot, 0, \cdot) & =\int_{0}^{A} g(a) z_{\lambda} d a+e^{-r t} \eta_{1} & & \text { in } Q_{T} .
\end{aligned}\right.
$$

Multiplying the first equation of system (2.7) by $z_{\lambda}$ and integrating by parts over $Q$, then using the fact that $\mu \geq 0$, we obtain

$$
\left\|\nabla z_{\lambda}\right\|_{L^{2}(Q)}^{2}+r\left\|z_{\lambda}\right\|_{L^{2}(Q)}^{2} \leq \frac{1}{2}\left\|z_{\lambda}(\cdot, 0, \cdot)\right\|_{L^{2}\left(Q_{T}\right)}^{2} .
$$

Since

$$
\begin{aligned}
\left\|z_{\lambda}(\cdot, 0, \cdot)\right\|_{L^{2}\left(Q_{T}\right)}^{2} & \leq 2\|g\|_{L^{2}(0, A)}^{2}\left\|z_{\lambda}\right\|_{L^{2}(Q)}^{2} \\
& +2\|h\|_{L^{2}(0, A)}^{2}\|y(v+\lambda w, g+\lambda h)-y(v, g)\|_{L^{2}\left(U ; H_{0}^{1}(\Omega)\right)}^{2},
\end{aligned}
$$


it follows from (2.8) that

$$
\begin{aligned}
& \left\|\nabla z_{\lambda}\right\|_{L^{2}(Q)}^{2}+\left(r-\|g\|_{L^{2}(0, A)}^{2}\right)\left\|z_{\lambda}\right\|_{L^{2}(Q)}^{2} \leq \\
& \|h\|_{L^{2}(0, A)}^{2}\|y(v+\lambda w, g+\lambda h)-y(v, g)\|_{L^{2}\left(U ; H_{0}^{1}(\Omega)\right)}^{2} .
\end{aligned}
$$

Choosing $r$ such that $r>\|g\|_{L^{2}(0, A)}^{2}$, we deduce

$$
\left\|z_{\lambda}\right\|_{L^{2}\left(U ; H_{0}^{1}(\Omega)\right)} \leq\|h\|_{L^{2}(0, A)}\|y(v+\lambda w, g+\lambda h)-y(v, g)\|_{L^{2}\left(U ; H_{0}^{1}(\Omega)\right)} .
$$

Hence,

$$
\left\|y_{\lambda}\right\|_{L^{2}\left(U ; H_{0}^{1}(\Omega)\right)} \leq e^{r T}\|h\|_{L^{2}(0, A)}\|y(v+\lambda w, g+\lambda h)-y(v, g)\|_{L^{2}(Q)} .
$$

Passing to the limit in this latter identity when $\lambda \rightarrow 0$ and using the fact that the function $(v, g) \mapsto y(v, g)$ is continuous, it follows that $y_{\lambda} \rightarrow 0$ strongly in $L^{2}\left(U ; H_{0}^{1}(\Omega)\right)$. This means that $\left(\bar{y}_{\lambda}\right)$ converges to $\bar{y}$ strongly in $L^{2}\left(U ; H_{0}^{1}(\Omega)\right)$ as $\lambda \rightarrow 0$.

Proposition 2.3. The mapping

$$
\begin{aligned}
\frac{\partial y}{\partial g}(\cdot, g): L^{2}\left(Q_{\omega}\right) & \rightarrow \mathcal{L}\left(L^{2}(0, A) ; L^{2}\left(U ; H_{0}^{1}(\Omega)\right)\right. \\
v & \mapsto \frac{\partial y}{\partial g}(v, g),
\end{aligned}
$$

is continuous.

Proof. From Proposition 2.2, we have that $\bar{y}(h)=\frac{\partial y}{\partial g}(v, g)(h)$ is a solution to

$$
\left\{\begin{aligned}
L \bar{y}(h) & =0 & & \text { in } Q, \\
\bar{y}(h) & =0 & & \text { on } \Sigma, \\
\bar{y}(h)(0, \cdot, \cdot) & =0 & & \text { in } Q_{A}, \\
\bar{y}(h)(\cdot, 0, \cdot) & =\int_{0}^{A} g(a) \bar{y}(h) d a+\int_{0}^{A} h(a) y(t, a, x ; v, g) d a & & \text { in } Q_{T} .
\end{aligned}\right.
$$

Let $v_{1}, v_{2} \in L^{2}\left(Q_{\omega}\right)$. Set $\bar{y}_{1}(h)=\frac{\partial y}{\partial g}\left(v_{1}, g\right)(h), \bar{y}_{2}(h)=\frac{\partial y}{\partial g}\left(v_{2}, g\right)(h)$ and take $\bar{z}(h)=$ $e^{-r t}\left(\bar{y}_{1}(h)-\bar{y}_{2}(h)\right), r>0$. It then follows that $\bar{z}(h)$ is a solution to problem

where for $(t, x) \in Q_{T}$,

$$
\eta_{4}(t, x)=\int_{0}^{A} h(a)\left(y\left(t, a, x ; v_{1}, g\right)-y\left(t, a, x ; v_{2}, g\right)\right) d a .
$$

Multiplying the first equation of system $(2.11)$ by $\bar{z}(h)$ and integrating by parts over $Q$, we obtain

$$
\begin{aligned}
& \frac{1}{2}\|\bar{z}(h)(T, \cdot, \cdot)\|_{L^{2}\left(Q_{A}\right)}^{2}+\frac{1}{2}\|\bar{z}(h)(\cdot, A, \cdot)\|_{L^{2}\left(Q_{T}\right)}^{2}-\frac{1}{2}\|\bar{z}(h)(\cdot, 0, \cdot)\|_{L^{2}\left(Q_{T}\right)}^{2} \\
& +\|\nabla \bar{z}(h)\|_{L^{2}(Q)}^{2}+\int_{Q}(r+\mu) \bar{z}(h)=0 .
\end{aligned}
$$


Observing

$$
\begin{aligned}
\|\bar{z}(h)(\cdot, 0, \cdot)\|_{L^{2}\left(Q_{T}\right)}^{2} & \leq 2\|g\|_{L^{2}(0, A)}^{2}\|\bar{z}(h)\|_{L^{2}(Q)}^{2} \\
& +\|h\|_{L^{2}(0, A)}^{2}\left\|y\left(v_{1}, g\right)-y\left(v_{2}, g\right)\right\|_{L^{2}\left(U ; H_{0}^{1}(\Omega)\right)}^{2}
\end{aligned}
$$

and choosing in (2.13) $r$ such that $r>\|g\|_{L^{2}(0, A)}^{2}$, we deduce

$$
\|\bar{z}(h)\|_{L^{2}\left(U ; H_{0}^{1}(\Omega)\right)} \leq\|h\|_{L^{2}(0, A)}\left\|y\left(v_{1}, g\right)-y\left(v_{2}, g\right)\right\|_{L^{2}\left(U ; H_{0}^{1}(\Omega)\right)} .
$$

Therefore,

$$
\left\|\bar{y}_{1}(h)-\bar{y}_{2}(h)\right\|_{L^{2}\left(U ; H_{0}^{1}(\Omega)\right)} \leq e^{r T}\|h\|_{L^{2}(0, A)}\left\|y\left(v_{1}, g\right)-y\left(v_{2}, g\right)\right\|_{L^{2}\left(U ; H_{0}^{1}(\Omega)\right)},
$$

from which we deduce

$$
\begin{aligned}
\left\|\bar{y}_{1}-\bar{y}_{2} \mid\right\| & =\sup _{\substack{h \in L^{2}(0, A),\|h\| \leq 1\\
}}\left\|\bar{y}_{1}(h)-\bar{y}_{2}(h)\right\|_{L^{2}\left(U ; H_{0}^{1}(\Omega)\right)} \\
& \leq e^{r T}\left\|y\left(v_{1}, g\right)-y\left(v_{2}, g\right)\right\|_{L^{2}\left(U ; H_{0}^{1}(\Omega)\right)},
\end{aligned}
$$

where $\||\cdot|\|$ stands for the norm in $\mathcal{L}\left(L^{2}(0, A) ; L^{2}\left(U ; H_{0}^{1}(\Omega)\right)\right)$. This leads us to

$$
\left\|\left|\frac{\partial y}{\partial g}\left(v_{1}, g\right)-\frac{\partial y}{\partial g}\left(v_{2}, g\right)\left\|\mid \leq e^{r T}\right\| y\left(v_{1}, g\right)-y\left(v_{2}, g\right) \|_{L^{2}\left(U ; H_{0}^{1}(\Omega)\right)} .\right.\right.
$$

Passing to the limit in this latter inequality when $v_{1} \rightarrow v_{2}$ while using Proposition 2.2 , we obtain that $\frac{\partial y}{\partial g}\left(v_{1}, g\right)$ converges to $\frac{\partial y}{\partial g}\left(v_{2}, g\right)$ in $\mathcal{L}\left(L^{2}(0, A) ; L^{2}\left(U ; H_{0}^{1}(\Omega)\right)\right)$.

3. Resolution of the optimization problem (1.5). In this section, we are concerned with the optimization problem (1.5). As the Low-regret and No-regret notion introduced by Lions [1] uses the decomposition of the solution of (1.1) on the form $y(v, g)=y(v, 0)+\varphi(g)$ where $y(v, 0)$ is solution of (1.1) with $g=0$ and $\varphi(g)$ a function depending of $g$, this decomposition is no longer valid because the map $g \mapsto y(v, g)$ from $L^{2}(0, A)$ to $L^{2}\left(U ; H_{0}^{1}(\Omega)\right)$ is non-linear. Thus using the regularity results of $y$ proven in Proposition 2.1 and Proposition 2.2, we replace the cost function defined in (1.4) by its linearized form with respect to $g$. We thus consider as in [17] the new cost-function

$$
J_{1}(v, g)=J(v, 0)+\frac{\partial J}{\partial g}(v, 0)(g) .
$$

Then, we consider the following new optimization problem:

$$
\inf _{v \in L^{2}\left(Q_{\omega}\right)} \sup _{g \in L^{2}(0, A)}\left(J_{1}(v, g)-J_{1}(0, g)\right) .
$$

Let $y(v, 0) \in L^{2}\left(U ; H_{0}^{1}(\Omega)\right)$ be the solution of

$$
\left\{\begin{aligned}
L y(v, 0) & =f+v \chi_{\omega} & & \text { in } \quad Q, \\
y & =0 & & \text { on } \Sigma, \\
y(0, \cdot, \cdot) & =y^{0} & & \text { in } Q_{A}, \\
y(\cdot, 0, \cdot) & =0 & & \text { in } Q_{T} .
\end{aligned}\right.
$$

Then we have the following result. 
Proposition 3.1. For any $(v, g) \in L^{2}\left(Q_{\omega}\right) \times L^{2}(0, A)$, the following equality holds:

where $J$ is the cost function defined in (1.4) and

$$
\frac{\partial J}{\partial g}(v, 0)(g)=\lim _{t \rightarrow 0} \frac{J(v, t g)-J(v, 0)}{t} .
$$

Proof. Observing on the one hand

$$
\begin{aligned}
J(v, t g) & =\left\|y(v, t g)-z_{d}\right\|_{L^{2}(Q)}^{2}+N\|v\|_{L^{2}\left(Q_{\omega}\right)}^{2} \\
& =J(v, 0)+\|y(v, t g)-y(v, 0)\|_{L^{2}(Q)}^{2} \\
& +2 \int_{Q}(y(v, t g)-y(v, 0))\left(y(v, 0)-z_{d}\right) d t d a d x,
\end{aligned}
$$

and on the other hand

$$
\frac{\partial J}{\partial g}(v, 0)(g)=\lim _{t \rightarrow 0} \frac{J(v, t g)-J(v, 0)}{t},
$$

using Proposition 2.2, we obtain that

$$
\frac{\partial J}{\partial g}(v, 0)(g)=2 \int_{Q}\left(\frac{\partial y}{\partial g}(v, 0)(g)\right)\left(y(v, 0)-z_{d}\right) d t d a d x .
$$

So, $J_{1}(v, g)=J(v, 0)+2 \int_{Q}\left(\frac{\partial y}{\partial g}(v, 0)(g)\right)\left(y(v, 0)-z_{d}\right) d t d a d x$.

Proposition 3.2. For any $(v, g) \in L^{2}\left(Q_{\omega}\right) \times L^{2}(0, A)$, we have

$$
J_{1}(v, g)-J_{1}(0, g)=J(v, 0)-J(0,0)+2 \int_{0}^{A} S(a ; v) g(a) d a,
$$

where for any $a \in(0, A)$,

$$
S(a ; v)=\int_{Q_{T}}[y(t, a, x ; v, 0) \xi(v)(t, 0, x)-y(t, a, x ; 0,0) \xi(0)(t, 0, x)] d t d x
$$

with $\xi(v)$, a solution to

$$
\left\{\begin{aligned}
L^{*} \xi(v) & =y(v, 0)-z_{d} & & \text { in } Q \\
\xi(v) & =0 & & \text { on } \Sigma, \\
\xi(v)(T, \cdot, \cdot) & =0 & & \text { in } Q_{A}, \\
\xi(v)(\cdot, A, \cdot) & =0 & & \text { in } Q_{T} .
\end{aligned}\right.
$$

Proof. In view of (3.4), we have

$$
\begin{aligned}
J_{1}(v, g)-J_{1}(0, g) & =J(v, 0)-J(0,0) \\
& +2 \int_{Q}\left(\frac{\partial y}{\partial g}(v, 0)(g)\right)\left(y(v, 0)-z_{d}\right) d t d a d x \\
& -\int_{Q}\left(\frac{\partial y}{\partial g}(0,0)(g)\right)\left(y(0,0)-z_{d}\right) d t d a d x
\end{aligned}
$$


191 From Proposition 2.2, we have that $\bar{y}(g)=\frac{\partial y}{\partial g}(v, 0)(g)$ is the solution to

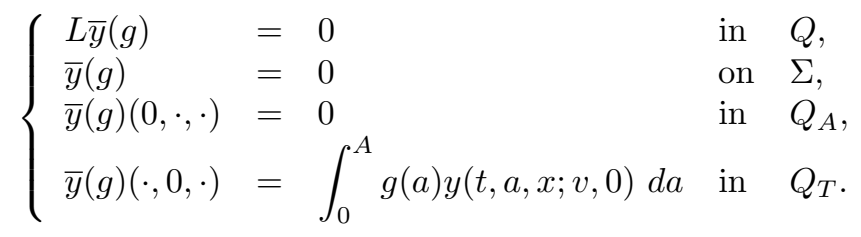

So, if we multiply the first equation of $(3.9)$ by $\xi(v)$ and integrate by parts over $Q$, we get

$$
-\int_{Q} g(a) y(t, a, x ; v, 0) \xi(v)(t, 0, x) d t d a d x+\int_{Q} \bar{y}(g)\left(y(v, 0)-z_{d}\right) d t d a d x=0,
$$

which can be rewritten as

$$
\begin{aligned}
& \int_{Q}\left(\frac{\partial y}{\partial g}(v, 0)(g)\right)\left(y(v, 0)-z_{d}\right) d t d a d x= \\
& \int_{Q} g(a) y(t, a, x ; v, 0) \xi(v)(t, 0, x) d t d a d x
\end{aligned}
$$

We also have

$$
\begin{aligned}
& \int_{Q}\left(\frac{\partial y}{\partial g}(0,0)(g)\right)\left(y(0,0)-z_{d}\right) d t d a d x= \\
& \int_{Q} g(a) y(t, a, x ; 0,0) \xi(0)(t, 0, x) d t d a d x
\end{aligned}
$$

Using (3.8), (3.10) and (3.11), it follows that

$$
\begin{aligned}
& J_{1}(v, g)-J_{1}(0, g)=J(v, 0)-J(0,0)+ \\
& 2 \int_{0}^{A} g(a) \int_{Q_{T}}[y(t, a, x ; v, 0) \xi(v)(t, 0, x)-y(t, a, x ; 0,0) \xi(0)(t, 0, x)] d a d t d x \\
& =J(v, 0)-J(0,0)+2 \int_{0}^{A} S(a ; v) g(a) d a .
\end{aligned}
$$

Lemma 3.3. Let $\xi(v)$ be the solution of problem (3.7). Then the application $v \mapsto$ $\xi(v)$ is continuous from $L^{2}\left(Q_{\omega}\right)$ onto $L^{2}\left(U ; H_{0}^{1}(\Omega)\right)$.

Proof. Let $v_{1}, v_{2} \in L^{2}\left(Q_{\omega}\right)$, and define $\bar{\xi}=\xi\left(v_{1}\right)-\xi\left(v_{2}\right)$. Then $\bar{\xi}$ is the solution to problem

$$
\left\{\begin{aligned}
L^{*} \bar{\xi} & =y\left(v_{1}, 0\right)-y\left(v_{2}, 0\right) & & \text { in } Q, \\
\bar{\xi} & =0 & & \text { on } \Sigma, \\
\bar{\xi}(T, \cdot, \cdot) & =0 & & \text { in } Q_{A}, \\
\bar{\xi}(\cdot, A, \cdot) & =0 & & \text { in } Q_{T} .
\end{aligned}\right.
$$

By setting $z=e^{-r t} \bar{\xi}$, it follows that $z$ solves

$$
\left\{\begin{aligned}
L^{*} z+r z & =\left(y\left(v_{1}, 0\right)-y\left(v_{2}, 0\right)\right) e^{-r t} & & \text { in } Q, \\
z & =0 & & \text { on } \Sigma, \\
z(T, \cdot, \cdot) & =0 & & \text { in } Q_{A} \\
z(\cdot, A, \cdot) & =0 & & \text { in } Q_{T} .
\end{aligned}\right.
$$


If we multiply the first equation of system (3.14) by $z$ and integrating by parts over $Q$, we get

$$
\begin{aligned}
& \frac{1}{2}\|z(0, \cdot, \cdot)\|_{L^{2}\left(Q_{A}\right)}^{2}+\frac{1}{2}\|z(\cdot, 0, \cdot)\|_{L^{2}\left(Q_{T}\right)}^{2}+\|\nabla z\|_{L^{2}(Q)}^{2}+\int_{Q}(r+\mu) z^{2} d t d a d x= \\
& \int_{Q}\left(y\left(v_{1}, 0\right)-y\left(v_{2}, 0\right)\right) z e^{-r t} d t d a d x .
\end{aligned}
$$

It then follows

$\frac{1}{2}\|z(\cdot, 0, \cdot)\|_{L^{2}\left(Q_{T}\right)}^{2}+\|\nabla z\|_{L^{2}(Q)}^{2}+r\|z\|_{L^{2}(Q)}^{2} \leq \frac{1}{2}\left\|y\left(v_{1}, 0\right)-y\left(v_{2}, 0\right)\right\|_{L^{2}(Q)}^{2}+\frac{1}{2}\|z\|_{L^{2}(Q)}^{2}$.

Taking $r=\frac{1}{2}$ in this latter identity yields

$$
\frac{1}{2}\|z(\cdot, 0, \cdot)\|_{L^{2}\left(Q_{T}\right)}^{2}+\frac{1}{2}\|z\|_{L^{2}\left(U ; H_{0}^{1}(\Omega)\right)}^{2} \leq \frac{1}{2}\left\|y\left(v_{1}, 0\right)-y\left(v_{2}, 0\right)\right\|_{L^{2}(Q)}^{2} .
$$

Thus,

$$
\|\bar{\xi}(\cdot, 0, \cdot)\|_{L^{2}\left(Q_{T}\right)}^{2}+\|\bar{\xi}\|_{L^{2}\left(U ; H_{0}^{1}(\Omega)\right)}^{2} \leq e^{T}\left\|y\left(v_{1}, 0\right)-y\left(v_{2}, 0\right)\right\|_{L^{2}(Q)},
$$

from which we deduce

$$
\|\bar{\xi}\|_{L^{2}\left(U ; H_{0}^{1}(\Omega)\right)} \leq e^{T / 2}\left\|y\left(v_{1}, 0\right)-y\left(v_{2}, 0\right)\right\|_{L^{2}(Q)} .
$$

Using Proposition 2.1, while passing to limit in this latter inequality when $v_{1} \rightarrow v_{2}$, we obtain that $\bar{\xi} \rightarrow 0$ strongly in $L^{2}\left(U ; H_{0}^{1}(\Omega)\right)$. This means that $\xi\left(v_{1}\right) \rightarrow \xi\left(v_{2}\right)$ strongly in $L^{2}\left(U ; H_{0}^{1}(\Omega)\right)$ as $v_{1} \rightarrow v_{2}$.

Proposition 3.4. Let $S(\cdot ; v)$ be the function defined in (3.6). Then the map $v \mapsto S(\cdot ; v)$ is continuous form $L^{2}\left(Q_{\omega}\right)$ onto $L^{2}(0, A)$.

Proof. Let $v_{1}$ and $v_{2}$. Then in view of (3.6),

$$
\begin{aligned}
S\left(a ; v_{1}\right)-S\left(a ; v_{2}\right) & =\int_{Q_{T}}\left(y\left(t, a, x ; v_{1}, 0\right)-y\left(t, a, x ; v_{2}, 0\right)\right) \xi\left(v_{1}\right)(t, 0, x) d t d x \\
& -\int_{Q_{T}} y\left(t, a, x ; v_{2}, 0\right)\left(\xi\left(v_{2}\right)(t, 0, x)-\xi\left(v_{1}\right)(t, 0, x)\right) d t d x
\end{aligned}
$$

Using the Cauchy Schwarz inequality, we have

$$
\begin{aligned}
& \left|S\left(a ; v_{1}\right)-S\left(a ; v_{2}\right)\right| \leq\left\|y\left(., a, . ; v_{1}, 0\right)-y\left(., a, . ; v_{2}, 0\right)\right\|_{L^{2}\left(Q_{T}\right)}\left\|\xi\left(v_{1}\right)(., 0, .)\right\|_{L^{2}\left(Q_{T}\right)} \\
& +\left\|y\left(., a, . ; v_{2}, 0\right)\right\|_{L^{2}\left(Q_{T}\right)}\left\|\xi\left(v_{2}\right)(., 0, .)-\xi\left(v_{1}\right)(., 0, .)\right\|_{L^{2}\left(Q_{T}\right)} .
\end{aligned}
$$

Observing on the one hand that $\xi\left(v_{2}\right)-\xi\left(v_{1}\right)$ is solution of (3.13), and, on the other hand that, in view of (3.15),

$$
\left\|\xi\left(v_{2}\right)(., 0, .)-\xi\left(v_{1}\right)(., 0, .)\right\|_{L^{2}\left(Q_{T}\right)}^{2} \leq e^{T / 2}\left\|y\left(v_{1}, 0\right)-y\left(v_{2}, 0\right)\right\|_{L^{2}(Q)},
$$

we have

$$
\begin{aligned}
& \left|S\left(a ; v_{1}\right)-S\left(a ; v_{2}\right)\right| \leq\left\|y\left(., a, . ; v_{1}, 0\right)-y\left(., a, . ; v_{2}, 0\right)\right\|_{L^{2}\left(Q_{T}\right)}\left\|\xi\left(v_{1}\right)(., 0, .)\right\|_{L^{2}\left(Q_{T}\right)} \\
& +e^{T / 2}\left\|y\left(., a, . ; v_{2}, 0\right)\right\|_{L^{2}\left(Q_{T}\right)}\left\|y\left(v_{1}, 0\right)-y\left(v_{2}, 0\right)\right\|_{L^{2}(Q)} .
\end{aligned}
$$


Hence,

$$
\begin{aligned}
& \int_{0}^{A}\left|S\left(a ; v_{1}\right)-S\left(a ; v_{2}\right)\right|^{2} d a \leq \\
& 2\left\|y\left(., a, . ; v_{1}, 0\right)-y\left(., a, . ; v_{2}, 0\right)\right\|_{L^{2}(Q)}^{2}\left\|\xi\left(v_{1}\right)(., 0, .)\right\|_{L^{2}\left(Q_{T}\right)}^{2}+ \\
& 2 e^{T}\left\|y\left(., a, . ; v_{2}, 0\right)\right\|_{L^{2}(Q)}^{2}\left\|y\left(v_{1}, 0\right)-y\left(v_{2}, 0\right)\right\|_{L^{2}(Q)}^{2} .
\end{aligned}
$$

It then follows from Poincaré inequality,

$$
\begin{aligned}
& \left\|S\left(a ; v_{1}\right)-S\left(a ; v_{2}\right)\right\|_{L^{2}(0, A)} \leq \\
& C(\Omega)\left\|y\left(., a, . ; v_{1}, 0\right)-y\left(., a, . ; v_{2}, 0\right)\right\|_{L^{2}\left(U ; H_{0}^{1}(\Omega)\right)}\left\|\xi\left(v_{1}\right)(., 0, .)\right\|_{L^{2}\left(Q_{T}\right)}+ \\
& C(\Omega) e^{T / 2}\left\|y\left(., a, . ; v_{2}, 0\right)\right\|_{L^{2}(Q)}\left\|y\left(v_{1}, 0\right)-y\left(v_{2}, 0\right)\right\|_{L^{2}\left(U ; H_{0}^{1}(\Omega)\right)},
\end{aligned}
$$

where $C(\Omega)>0$ is a constant depending on $\Omega$. In view of Proposition 2.1, it follows that $S\left(\cdot, v_{1}\right) \rightarrow S\left(\cdot, v_{2}\right)$ as $v_{1} \rightarrow v_{2}$.

The following Lemma will be useful to prove the existence of the No-regret and Lowregret controls.

Lemma 3.5. Let $S(., v)$ be defined as in (3.6) for any $a \in L^{2}(0, A)$. For any $\gamma>0$, we consider the sequences $y^{\gamma}=y\left(t, a, x ; u^{\gamma}, 0\right)$ and $\xi\left(u^{\gamma}\right)$, respectively, solutions of (3.3) and (3.7) with $v=u^{\gamma}$. Assume that there exists $C>0$ independent of $\gamma$ such that

$$
\left\|S\left(., u^{\gamma}\right)\right\|_{L^{2}(0, A)}<C .
$$

Assume also that $\hat{u} \in L^{2}\left(Q_{\omega}\right), \hat{\xi}(., 0,.) \in L^{2}\left(Q_{T}\right)$ and $\hat{y}=y(t, a, x ; \hat{u}, 0) \in L^{2}\left(U ; H_{0}^{1}(\Omega)\right.$ solution of (3.3), such that

$$
\begin{array}{rrr}
u^{\gamma} \rightarrow & \hat{u} \text { weakly in } & L^{2}(U \times \omega), \\
y^{\gamma} \rightarrow \hat{y}=y(t, a, x ; \hat{u}, 0) & \text { weakly in } L^{2}\left(U, H_{0}^{1}(\Omega)\right), \\
\xi\left(u^{\gamma}\right)(., 0, .) \rightarrow & \hat{\xi}(., 0, .) \text { weakly in } & L^{2}\left(Q_{T}\right) .
\end{array}
$$

Then we have

$$
S\left(a ; u^{\gamma}\right) \rightarrow S(a ; \hat{u}) \text { weakly in } \mathcal{D}^{\prime}(0, A) .
$$

Proof. Let $\mathcal{D}((0, A))$ be the set of $\mathcal{C}^{\infty}$ function with compact support on $(0, A)$. Set for any $\phi \in \mathcal{D}((0, A))$

$$
z^{\gamma}(t, x)=\int_{0}^{A} y\left(t, a, x ; u^{\gamma}, 0\right) \phi(a) d a,(t, x) \in Q_{T} .
$$

Then, in view of (3.16b), there exists a constant $C>0$ independent of $\gamma$ such that

$$
\left\|z^{\gamma}\right\|_{L^{2}\left(Q_{T}\right)} \leq\left\|y^{\gamma}\right\|_{L^{2}(Q)}\|\phi\|_{L^{2}(0, A)} \leq C .
$$

Consequently, there exists $z \in L^{2}\left(Q_{T}\right)$ such that

$$
z^{\gamma} \rightarrow z \text { weakly in } L^{2}\left(Q_{T}\right) .
$$

Moreover, using (3.17) we have that,

$$
\int_{Q_{T}} z^{\gamma}(t, x) \psi(t, x) d x d t=\int_{Q_{T}} \int_{0}^{A} y\left(t, a, x ; u^{\gamma}, 0\right) \phi(a) \psi(t, x) d x d t d a, \forall \psi \in \mathcal{D}\left(Q_{T}\right),
$$


which passing to the limit when $\gamma \rightarrow 0$ while using (3.16b) gives $\lim _{\gamma \rightarrow 0} \int_{Q_{T}} z^{\gamma}(t, x) z(t, x) d x d t=\int_{Q_{T}} \int_{0}^{A} y(t, a, x ; \hat{u}, 0) \phi(a) \psi(t, x) d x d t d a \forall \psi \in \mathcal{D}\left(Q_{T}\right)$.

This means that

$$
z^{\gamma} \rightarrow \int_{0}^{A} y(t, a, x ; \hat{u}, 0) \phi(a) d a \text { weakly in } \mathcal{D}^{\prime}\left(Q_{T}\right) .
$$

$$
z(t, x)=\int_{0}^{A} y(t, a, x ; \hat{u}, 0) \phi(a) d a,(t, x) \in Q_{T} .
$$

Because $y^{\gamma}=y\left(t, a, x ; u^{\gamma}, 0\right)$ solves (3.3) with $v=u^{\gamma}$, we have that $z^{\gamma}$ solves

$$
\left\{\begin{array}{llll}
\frac{\partial z^{\gamma}}{\partial t}-\Delta z^{\gamma} & =k^{\gamma} & & \text { in } \quad Q_{T}, \\
z^{\gamma} & =0 & & \text { on } \quad(0, T) \times \Gamma, \\
z^{\gamma}(0) & =\int_{0}^{A} y^{0}(a, x) \phi(a) d a & & \text { in } \Omega,
\end{array}\right.
$$

where

$$
k^{\gamma}(t, x)=\int_{0}^{A}\left(f+u^{\gamma} \chi_{\omega}\right) \phi d a-\int_{0}^{A} \mu(a) y^{\gamma} \phi d a-\int_{0}^{A} \frac{\partial y^{\gamma}}{\partial a} \phi d a .
$$

Consequently, in view of (3.16a) and (3.16b), we have there exists a positive constant $C$ independent of $\gamma$ such that

$$
\begin{aligned}
& \left\|k^{\gamma}\right\|_{L^{2}\left(Q_{T}\right)} \leq\left(2\|f\|_{L^{2}(Q)}^{2}+2\left\|u^{\gamma}\right\|_{L^{2}\left(Q_{\omega}\right)}^{2}+\|\mu\|_{L^{\infty}(0, A)}^{2}\left\|y^{\gamma}\right\|_{L^{2}(Q)}^{2}\right)^{1 / 2}\|\phi\|_{L^{2}(0, A)}+ \\
& \left\|y^{\gamma}\right\|_{L^{2}(Q)}\left\|\frac{\partial \phi}{\partial a}\right\|_{L^{2}(0, A)} \leq C .
\end{aligned}
$$

It then follows that there is $C>0$, independent of $\gamma$, such that

$$
\begin{cases}\left\|z^{\gamma}\right\|_{L^{2}\left((0, T) ; H_{0}^{1}(\Omega)\right)} & \leq C, \\ \left\|\frac{\partial z^{\gamma}}{\partial t}\right\|_{L^{2}\left((0, T) ; H^{-1}(\Omega)\right)} & \leq C .\end{cases}
$$

Therefore, it follows from Aubin-Lions's Lemma that

$$
z^{\gamma} \rightarrow z \text { strongly in } L^{2}\left(Q_{T}\right)
$$

where

$$
z(t, x)=\int_{0}^{A} y(t, a, x ; \hat{u}, 0) \phi(a) d a,(t, x) \in Q_{T}
$$

because of (3.19).

Now in view of (3.6)

$$
S\left(a ; u^{\gamma}\right)=\int_{Q_{T}}\left[y\left(t, a, x ; u^{\gamma}, 0\right) \xi\left(u^{\gamma}\right)(t, 0, x)-y(t, a, x ; 0,0) \xi(0)(t, 0, x)\right] d t d x .
$$


Therefore for any $\phi \in \mathcal{D}(0, A)$,

$$
\begin{aligned}
& \int_{0}^{A} S\left(a ; u^{\gamma}\right) \phi(a) d a=\int_{Q_{T}} \int_{0}^{A}\left(y\left(t, a, x ; u^{\gamma}, 0\right) \phi(a) d a\right) \xi\left(u^{\gamma}\right)(t, 0, x) d t d a d x \\
& \text { - } \int_{Q} y(t, a, x ; 0,0) \xi(0)(t, 0, x) \phi(a) d t d a d x \\
& =\int_{Q_{T}}^{Q_{Q}} z^{\gamma}(t, x) \xi\left(u^{\gamma}\right)(t, 0, x) d t d a d x \\
& -\int_{Q}^{Q_{T}} y(t, a, x ; 0,0) \xi(0)(t, 0, x) \phi(a) d t d a d x
\end{aligned}
$$

Passing this latter identity to the limit while using (3.20), (3.19) and (3.16c), we obtain

$$
\begin{aligned}
& \int_{0}^{A} S\left(a ; u^{\gamma}\right) \phi(a) d a \rightarrow \int_{Q_{T}} z(t, x) \xi(\hat{u})(t, 0, x) d t d a d x \\
& -\quad \int_{Q} y(t, a, x ; 0,0) \xi(0)(t, 0, x) \phi(a) d t d a d x \\
& =\int_{Q_{T}} \int_{0}^{A}(y(t, a, x ; \hat{u}, 0) \phi(a) d a) \xi(\hat{u})(t, 0, x) d t d a d x \\
& \text { - } \int_{Q} y(t, a, x ; 0,0) \xi(0)(t, 0, x) \phi(a) d t d a d x \quad \forall \phi \in \mathcal{D}(0, A) \text {, }
\end{aligned}
$$

which in view of (3.6), proves

$$
S\left(a ; u^{\gamma}\right) \rightarrow S(a ; \hat{u}) \text { weakly in } \mathcal{D}^{\prime}(0, A) .
$$

From now on, we denote by $\mathcal{D}(\Theta)$ the set of $\mathcal{C}^{\infty}$ function with compact support on $\Theta$ and by $\mathcal{D}^{\prime}(\Theta)$, its dual.

3.1. Existence of No-regret control and Low-regret control. In view of (3.5), the optimization problem (3.2) is equivalent to the following problem:

$$
\inf _{v \in L^{2}\left(Q_{\omega}\right)} \sup _{g \in L^{2}(0, A)}\left[J(v, 0)-J(0,0)+2 \int_{0}^{A} S(a ; v) g(a) d a\right] .
$$

As $\int_{0}^{A} S(a ; v) g(a) d a$ is either equal to 0 or $+\infty$, we look for the control $v$ in the set,

$$
\mathcal{M}=\left\{v \in L^{2}\left(Q_{\omega}\right) ; \int_{0}^{A} S(a ; v) g(a) d a=0, \quad \forall g \in L^{2}(0, A)\right\}
$$

Note that the set $\mathcal{M}$ is strongly closed in $L^{2}\left(Q_{\omega}\right)$. Now, observing on the one hand that the application $v \mapsto J(v, 0)-J(0,0)$ is coercive on $L^{2}\left(Q_{\omega}\right)$, bounded below by $-J(0,0)$, and continuous because of Proposition 2.1, and on the other hand that the application $v \mapsto S(\cdot ; v)$ is continuous on $L^{2}\left(Q_{\omega}\right)$, using minimizing sequences and Lemma 3.5, we prove that there exists a No-regret control $\tilde{u}$ in $\mathcal{M}$ satisfying (3.21). We thus have proved the following result.

Lemma 3.6. There exists a solution $\tilde{u}$ of (3.21) in $\mathcal{M}$. 
As such a control $\tilde{u}$ is not easy to characterize, we consider for any $\gamma>0$, the relaxed optimization problem, which we refer to as the Low-regret-control problem:

$$
\inf _{v \in L^{2}\left(Q_{\omega}\right)} \sup _{g \in L^{2}(0, A)}\left[J(v, 0)-J(0,0)+2 \int_{0}^{A} S(a ; v) g(a) d a-\gamma\|g\|_{L^{2}(0, A)}^{2}\right] .
$$

Observing that

$$
\begin{gathered}
\sup _{g \in L^{2}(0, A)}\left[J(v, 0)-J(0,0)+2 \int_{0}^{A} S(a ; v) g(a) d a-\gamma\|g\|_{L^{2}(0, A)}^{2}\right]= \\
J(v, 0)-J(0,0)+2 \gamma \sup _{g \in L^{2}(0, A)}\left[\int_{0}^{A} \frac{S(a ; v)}{\gamma} g(a) d a-\frac{1}{2}\|g\|_{L^{2}(0, A)}^{2}\right],
\end{gathered}
$$

using Fenchel-Legendre transform (see [16]), we obtain that,

$$
2 \gamma \sup _{g \in L^{2}(0, A)}\left[\int_{0}^{A} \frac{S(a ; v)}{\gamma} g(a) d a-\frac{1}{2}\|g\|_{L^{2}(0, A)}^{2}\right]=\frac{1}{\gamma}\|S(\cdot ; v)\|_{L^{2}(0, A)}^{2}
$$

and (3.23) is equivalent to

$$
\inf _{v \in L^{2}\left(Q_{\omega}\right)} \mathcal{J}^{\gamma}(v)
$$

with

$$
\mathcal{J}^{\gamma}(v)=J(v, 0)-J(0,0)+\frac{1}{\gamma}\|S(\cdot ; v)\|_{L^{2}(0, A)}^{2} .
$$

Proposition 3.7. Let $\gamma>0$. Then there exists at least in $L^{2}\left(Q_{\omega}\right)$ a Low-regret control $u_{\gamma}$ solution of problem (3.24).

Proof. We have $\mathcal{J}^{\gamma}(v) \geq-J(0,0)$ and $\mathcal{J}^{\gamma}(0)=0$. Using minimizing sequences, Proposition 2.1, Proposition 3.4 and Lemma 3.5, we prove as for Lemma 3.6 that problem (3.24) has at least one solution $u_{\gamma} \in L^{2}\left(Q_{\omega}\right)$.

Remark 3.8. The uniqueness of $u_{\gamma} \in L^{2}\left(Q_{\omega}\right)$, solution of (3.24) is not guaranteed because the application $v \mapsto S(\cdot ; v)$ from $L^{2}\left(Q_{\omega}\right)$ to $L^{2}(0, A)$ is not necessarily strictly convex. Consequently, we are not sure that control $u_{\gamma}$ will converge to a No-regret control $\widetilde{u} \in \mathcal{M}$. So, in order to have a Low-regret control which will converge in $\mathcal{M}$, we adapt the cost function $\mathcal{J}^{\gamma}$ to a No-regret control $\widetilde{u}$.

3.2. Existence of the adapted low-regret control. Let $\widetilde{u}$ be a No-regret optimal control. For any $\gamma>0$, we define the adapted cost function $\widetilde{\mathcal{J}}^{\gamma}$ by:

$$
v \mapsto \widetilde{\mathcal{J}}^{\gamma}(v)=J(v, 0)-J(0,0)+\|v-\widetilde{u}\|_{L^{2}\left(Q_{\omega}\right)}^{2}+\frac{1}{\gamma}\|S(\cdot ; v)\|_{L^{2}(0, A)}^{2} .
$$

Then, we consider the following optimal control problem:

$$
\inf _{v \in L^{2}\left(Q_{\omega}\right)} \widetilde{\mathcal{J}}^{\gamma}(v)
$$


Proposition 3.9. Let $\gamma>0$. Then problem (3.27) has at least a solution $\widetilde{u}_{\gamma}$ in $L^{2}\left(Q_{\omega}\right)$.

Proof. One proceeds as for the proof of Proposition 3.7 using the fact that $v \mapsto$ $\widetilde{\mathcal{J}}^{\gamma}(v)$ is continuous on $L^{2}\left(Q_{\omega}\right)$ ( thanks to Proposition 2.1 and Proposition 3.4) and the fact that $\lim _{\|v\|_{L^{2}\left(Q_{\omega}\right)} \rightarrow+\infty} \mathcal{J}^{\gamma}(v)=+\infty$.

Proposition 3.10. Let $\widetilde{u}_{\gamma} \in L^{2}\left(Q_{\omega}\right)$ be a solution of (3.27). Then there exist $\widetilde{p}_{\gamma}=p\left(\widetilde{u}_{\gamma}\right) \in L^{2}\left(U ; H_{0}^{1}(\Omega)\right)$ and $\widetilde{q}_{\gamma}=q\left(\widetilde{u}_{\gamma}\right) \in L^{2}\left(U ; H_{0}^{1}(\Omega)\right)$ such that $\left\{\widetilde{y}_{\gamma}, \widetilde{\xi}_{\gamma}, \widetilde{p}_{\gamma}, \widetilde{q}_{\gamma}\right\}$ is a solution of the systems:

$$
\left\{\begin{aligned}
L \widetilde{y}_{\gamma} & =f+\widetilde{u}_{\gamma} \chi_{Q_{\omega}} & & \text { in } Q, \\
\widetilde{y}_{\gamma} & =0 & & \text { on } \Sigma, \\
\widetilde{y}_{\gamma}(0, \cdot, \cdot) & =y^{0} & & \text { in } Q_{A}, \\
\widetilde{y}_{\gamma}(\cdot, 0, \cdot) & =0 & & \text { in } Q_{T},
\end{aligned}\right.
$$

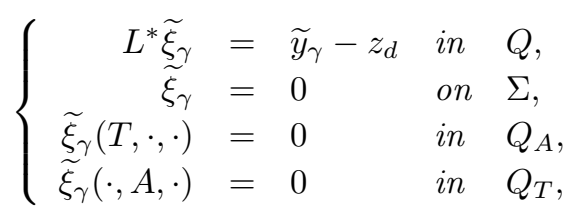

$$
\left\{\begin{array}{rlrl}
L \tilde{p}_{\gamma} & =0 & & \text { in } Q, \\
\widetilde{p}_{\gamma} & =0 & & \text { on } \Sigma, \\
\widetilde{p}_{\gamma}(0, \cdot, \cdot) & =0 & \text { in } Q_{A} \\
\widetilde{p}_{\gamma}(\cdot, 0, \cdot) & =\frac{1}{\sqrt{\gamma}} \int_{0}^{A} y\left(t, a, x ; \widetilde{u}_{\gamma}, 0\right) S\left(a ; \widetilde{u}_{\gamma}\right) d a & & \text { in } \quad Q_{T}
\end{array}\right.
$$

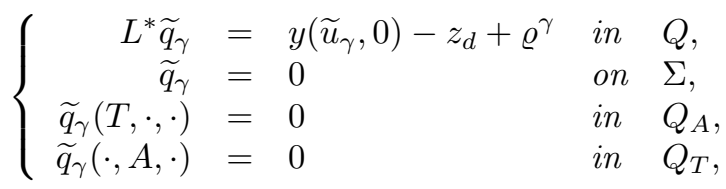

and

$$
(N+1) \widetilde{u}_{\gamma}-\widetilde{u}+\widetilde{q}_{\gamma}=0 \quad \text { in } Q_{\omega},
$$

where $\varrho^{\gamma}=\frac{1}{\sqrt{\gamma}} \widetilde{p}_{\gamma}+\frac{1}{\gamma} S\left(a ; \widetilde{u}_{\gamma}\right) \xi\left(\widetilde{u}_{\gamma}\right)(t, 0, x), \widetilde{y}_{\gamma}=y\left(\widetilde{u}_{\gamma}, 0\right)$ and $\widetilde{\xi}_{\gamma}=\xi\left(\widetilde{u}_{\gamma}\right)$.

Proof. We write the Euler-Lagrange optimality condition that characterizes $\widetilde{u}_{\gamma}$ :

$$
\lim _{\lambda \rightarrow 0} \frac{\widetilde{\mathcal{J}}^{\gamma}\left(\widetilde{u}_{\gamma}+\lambda w\right)-\widetilde{\mathcal{J}}^{\gamma}\left(\widetilde{u}_{\gamma}\right)}{\lambda}=0, \quad \forall w \in L^{2}\left(Q_{\omega}\right) .
$$

Using Proposition 2.2 and Proposition 3.2, we obtain after some calculations

$$
\begin{aligned}
0 & =\int_{Q}\left(\frac{\partial y}{\partial v}\left(\widetilde{u}_{\gamma}, 0\right)(w)\right)\left(y\left(\widetilde{u}_{\gamma}, 0\right)-z_{d}+\frac{1}{\gamma} \xi\left(\widetilde{u}_{\gamma}\right)(\cdot, 0, \cdot) S\left(\cdot ; \widetilde{u}_{\gamma}\right)\right) d t d a d x \\
& +\int_{Q_{\omega}}\left(\widetilde{u}_{\gamma}-\widetilde{u}\right) w d t d a d x+\int_{Q_{\omega}} N \widetilde{u}_{\gamma} w d t d a d x \\
& +\frac{1}{\gamma} \int_{Q} \frac{\partial \xi}{\partial v}\left(\widetilde{u}_{\gamma}\right)(w)(\cdot, 0, \cdot) y\left(\widetilde{u}_{\gamma}, 0\right) S\left(\cdot ; \widetilde{u}_{\gamma}\right) d t d a d x, \quad \forall w \in L^{2}\left(Q_{\omega}\right)
\end{aligned}
$$


293 where $\bar{y}(w)=\frac{\partial y}{\partial v}\left(\widetilde{u}_{\gamma}, 0\right)(w)$ and $\bar{\xi}=\frac{\partial \xi}{\partial v}\left(\widetilde{u}_{\gamma}\right)(w)$ are respectively solutions to

294

295

296

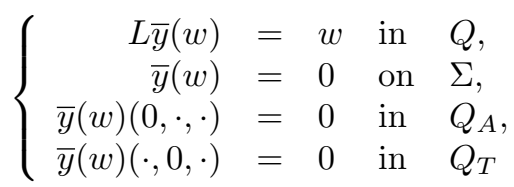

and

$$
\left\{\begin{aligned}
L^{*} \bar{\xi} & =\bar{y}(w) & & \text { in } \quad Q, \\
\bar{\xi} & =0 & & \text { on } \Sigma, \\
\bar{\xi}(T, \cdot, \cdot) & =0 & & \text { in } Q_{A}, \\
\bar{\xi}(\cdot, A, \cdot) & =0 & & \text { in } Q_{T} .
\end{aligned}\right.
$$

To interpret (3.33), we use the adjoint states $\widetilde{q}_{\gamma}$ and $\widetilde{p}_{\gamma}$ solutions of (3.31) and (3.30) respectively.

So if we multiply the first term of (3.34) by a function $\widetilde{q}_{\gamma}$ and the first equation of (3.35) by a function $\frac{1}{\sqrt{\gamma}} p_{\gamma}$, then integrate by parts over $Q$, we, respectively, obtain

$$
\begin{aligned}
& \int_{Q} \bar{y}(w)\left(y\left(\widetilde{u}_{\gamma}, 0\right)-z_{d}+\frac{1}{\sqrt{\gamma}} p_{\gamma}+\frac{1}{\gamma} S\left(a ; \widetilde{u}_{\gamma}\right) \xi\left(\widetilde{u}_{\gamma}\right)(t, 0, x)\right) d t d a d x= \\
& \int_{Q_{\omega}} w \widetilde{q}_{\gamma} d t d a d x,
\end{aligned}
$$

and

$$
\text { (3.37) } \frac{1}{\gamma} \int_{Q} \bar{\xi}(\cdot, 0, \cdot) y\left(t, a, x ; \widetilde{u}_{\gamma}, 0\right) S\left(a ; \widetilde{u}_{\gamma}\right) d t d a d x=\frac{1}{\sqrt{\gamma}} \int_{Q} \bar{y}(w) p_{\gamma} d t d a d x .
$$

Combining (3.36), (3.37) and (3.33), we have

$$
\int_{Q_{\omega}}\left((N+1) \widetilde{u}_{\gamma}-\widetilde{u}+\widetilde{q}_{\gamma}\right) w d t d a d x=0, \quad \forall w \in L^{2}\left(Q_{\omega}\right),
$$

which implies that

$$
(N+1) \widetilde{u}_{\gamma}-\widetilde{u}+\widetilde{q}_{\gamma}=0 \text { in } Q_{\omega} .
$$

Proposition 3.11. Let $\widetilde{u}_{\gamma} \in L^{2}\left(Q_{\omega}\right)$ be a solution of (3.27). Let also $\widetilde{y}_{\gamma}, \widetilde{\xi}_{\gamma}, \widetilde{p}_{\gamma}$ and $\widetilde{q}_{\gamma}$ be such that (3.28)-(3.32) hold true. Then we have following estimations:

$$
\left\|\widetilde{u}_{\gamma}\right\|_{L^{2}\left(Q_{\omega}\right)} \leq C\left(N,\|\widetilde{u}\|_{L^{2}\left(Q_{\omega}\right)},\left\|y^{0}\right\|_{L^{2}\left(Q_{A}\right)},\|f\|_{L^{2}(Q)},\left\|z_{d}\right\|_{L^{2}(Q)}\right),
$$

(3.40) $\frac{1}{\sqrt{\gamma}}\left\|S\left(\cdot ; \widetilde{u}_{\gamma}\right)\right\|_{L^{2}(0, A)} \leq C\left(\|\widetilde{u}\|_{L^{2}\left(Q_{\omega}\right)},\left\|y^{0}\right\|_{L^{2}\left(Q_{A}\right)},\|f\|_{L^{2}(Q)},\left\|z_{d}\right\|_{L^{2}(Q)}\right)$,

(3.41) $\quad\left\|S\left(\cdot ; \widetilde{u}_{\gamma}\right)\right\|_{L^{2}(0, A)} \leq \sqrt{\gamma} C\left(\|\widetilde{u}\|_{L^{2}\left(Q_{\omega}\right)},\left\|y^{0}\right\|_{L^{2}\left(Q_{A}\right)},\|f\|_{L^{2}(Q)},\left\|z_{d}\right\|_{L^{2}(Q)}\right)$,

$(3.42)\left\|\widetilde{y}_{\gamma}\right\|_{L^{2}\left(U ; H_{0}^{1}(\Omega)\right)} \leq C\left(N, T,\|\widetilde{u}\|_{L^{2}\left(Q_{\omega}\right)},\left\|y^{0}\right\|_{L^{2}\left(Q_{A}\right)},\|f\|_{L^{2}(Q)},\left\|z_{d}\right\|_{L^{2}(Q)}\right)$,

(3.43) $\left\|\widetilde{\xi}_{\gamma}\right\|_{L^{2}\left(U ; H_{0}^{1}(\Omega)\right)} \leq C\left(T,\|\widetilde{u}\|_{L^{2}\left(Q_{\omega}\right)},\left\|y^{0}\right\|_{L^{2}\left(Q_{A}\right)},\|f\|_{L^{2}(Q)},\left\|z_{d}\right\|_{L^{2}(Q)}\right)$,

(3.44) $\left\|\widetilde{\xi}_{\gamma}(., 0, .)\right\|_{L^{2}\left(Q_{T}\right)} \leq C\left(T,\|\widetilde{u}\|_{L^{2}\left(Q_{\omega}\right)},\left\|y^{0}\right\|_{L^{2}\left(Q_{A}\right)},\|f\|_{L^{2}(Q)},\left\|z_{d}\right\|_{L^{2}(Q)}\right)$,

(3.45) $\quad\left\|\widetilde{p}_{\gamma}(., 0, .)\right\|_{L^{2}\left(Q_{T}\right)} \leq C\left(\|\widetilde{u}\|_{L^{2}\left(Q_{\omega}\right)},\left\|y^{0}\right\|_{L^{2}\left(Q_{A}\right)},\|f\|_{L^{2}(Q)},\left\|z_{d}\right\|_{L^{2}(Q)}\right)$,

(3.46) $\left\|\widetilde{p}_{\gamma}\right\|_{L^{2}\left(U ; H_{0}^{1}(\Omega)\right)} \leq C\left(T,\|\widetilde{u}\|_{L^{2}\left(Q_{\omega}\right)},\left\|y^{0}\right\|_{L^{2}\left(Q_{A}\right)},\|f\|_{L^{2}(Q)},\left\|z_{d}\right\|_{L^{2}(Q)}\right)$,

(3.47) $\left\|\widetilde{q}_{\gamma}\right\|_{L^{2}\left(U ; H_{0}^{1}(\Omega)\right)} \leq C\left(N, T,\|\widetilde{u}\|_{L^{2}\left(Q_{\omega}\right)},\left\|y^{0}\right\|_{L^{2}\left(Q_{A}\right)},\|f\|_{L^{2}(Q)},\left\|z_{d}\right\|_{L^{2}(Q)}\right)$, 
where from now on, $C(X)$ to denote a positive constant whose value varies from a line to another but depends on $X$.

Proof. We proceed in three steps.

Step 1. We prove the estimations (3.39)-(3.43).

As $\widetilde{u}_{\gamma}$ is solution of (3.27), we can write

$$
\widetilde{\mathcal{J}}^{\gamma}\left(\widetilde{u}_{\gamma}\right) \leq \widetilde{\mathcal{J}}^{\gamma}(0)=\|\widetilde{u}\|_{L^{2}\left(Q_{\omega}\right)} .
$$

It then follows from the definition of $\tilde{\mathcal{J}}^{\gamma}$ and $J$ given respectively by (3.26) and (1.4) that,

$$
\begin{aligned}
& \left\|\widetilde{y}_{\gamma}-z_{d}\right\|_{L^{2}(Q)}^{2}+N\left\|\widetilde{u}_{\gamma}\right\|_{L^{2}\left(Q_{\omega}\right)}^{2}+\left\|\widetilde{u}_{\gamma}-\widetilde{u}\right\|_{L^{2}(Q)}^{2}+\frac{1}{\gamma}\left\|S\left(\cdot ; \widetilde{u}_{\gamma}\right)\right\|_{L^{2}(0, A)}^{2} \leq \\
& \|\widetilde{u}\|_{L^{2}\left(Q_{\omega}\right)}^{2}+\left\|y(0,0)-z_{d}\right\|_{L^{2}(Q)}^{2}=C\left(\|\widetilde{u}\|_{L^{2}\left(Q_{\omega}\right)},\left\|y^{0}\right\|_{L^{2}\left(Q_{A}\right)},\|f\|_{L^{2}(Q)},\left\|z_{d}\right\|_{L^{2}(Q)}\right) .
\end{aligned}
$$

Hence we deduce (3.39), (3.40), (3.41) and

$$
\left\|\widetilde{y}_{\gamma}-z_{d}\right\|_{L^{2}(Q)} \leq C\left(\|\widetilde{u}\|_{L^{2}\left(Q_{\omega}\right)},\left\|y^{0}\right\|_{L^{2}\left(Q_{A}\right)},\|f\|_{L^{2}(Q)},\left\|z_{d}\right\|_{L^{2}(Q)}\right) .
$$

Observing $\widetilde{y}_{\gamma}$ and $\widetilde{\xi}_{\gamma}$ are respectively solution of (3.28) and (3.29), proceeding as for $\bar{y}$ in pages $3-4$, we obtain that

$$
\left\|\widetilde{y}_{\gamma}\right\|_{L^{2}\left(U ; H_{0}^{1}(\Omega)\right)} \leq \frac{1}{\sqrt{2}} e^{2 T}\left(\left\|y_{0}\right\|_{L^{2}\left(Q_{A}\right)}+\|f\|_{L^{2}(Q)}+\left\|\widetilde{u}_{\gamma}\right\|_{L^{2}\left(Q_{\omega}\right)}\right)
$$

and

$$
\left\|\widetilde{\xi}_{\gamma}(., 0, .)\right\|_{L^{2}\left(Q_{T}\right)}+\left\|\widetilde{\xi}_{\gamma}\right\|_{L^{2}\left(U ; H_{0}^{1}(\Omega)\right)} \leq \frac{\sqrt{2}}{2} e^{2 T}\left\|\widetilde{y}_{\gamma}-z_{d}\right\|_{L^{2}(Q)},
$$

from which we, respectively, deduce (3.42), (3.43) and (3.44) because of (3.39) and (3.49).

Step 2. We prove the estimations (3.45) and (3.46).

To prove (3.45), we observe that

$$
\begin{aligned}
& \left|\frac{1}{\sqrt{\gamma}} \int_{0}^{A} y\left(t, a, x ; \widetilde{u}_{\gamma}, 0\right) S\left(a ; \widetilde{u}_{\gamma}\right) d a\right| \leq \\
& \frac{1}{\sqrt{\gamma}}\left\|S\left(. ; \widetilde{u}_{\gamma}\right)\right\|_{L^{2}(0, A)}\left(\int_{0}^{A} y\left(t, a, x ; \widetilde{u}_{\gamma}, 0\right)^{2} d a\right)^{1 / 2} .
\end{aligned}
$$

So using (3.40) and (3.49), we deduce

$$
\begin{aligned}
\int_{Q_{T}}\left|\frac{1}{\sqrt{\gamma}} \int_{0}^{A} y\left(t, a, x ; \widetilde{u}_{\gamma}, 0\right) S\left(a ; \widetilde{u}_{\gamma}\right) d a\right|^{2} d t d x & \leq \frac{1}{\gamma}\left\|S\left(. ; \widetilde{u}_{\gamma}\right)\right\|_{L^{2}(0, A)}^{2}\left\|\widetilde{y}_{\gamma}\right\|_{L^{2}(Q)}^{2} \\
& \leq C
\end{aligned}
$$

where $C=C\left(\|\widetilde{u}\|_{L^{2}\left(Q_{\omega}\right)},\left\|y^{0}\right\|_{L^{2}\left(Q_{A}\right)},\|f\|_{L^{2}(Q)},\left\|z_{d}\right\|_{L^{2}(Q)}\right)>0$. This means

$$
\left\|p_{\gamma}(\cdot, 0, \cdot)\right\|_{L^{2}(Q)} \leq C\left(\|\widetilde{u}\|_{L^{2}\left(Q_{\omega}\right)},\left\|y^{0}\right\|_{L^{2}\left(Q_{A}\right)},\|f\|_{L^{2}(Q)},\left\|z_{d}\right\|_{L^{2}(Q)}\right) .
$$

Since $p_{\gamma}$ is solution of (3.30), proceeding as for $\bar{y}$ in pages $3-4$ while using (3.45), we obtain

$$
\left\|\widetilde{p}_{\gamma}\right\|_{L^{2}\left(U ; H_{0}^{1}(\Omega)\right)} \leq \frac{1}{\sqrt{2}} e^{2 T}\left(\left\|y_{0}\right\|_{L^{2}\left(Q_{A}\right)}+\|f\|_{L^{2}(Q)}+\left\|\widetilde{u}_{\gamma}\right\|_{L^{2}\left(Q_{\omega}\right)}\right) .
$$


Step 3. We prove (3.47).

We observe that $\widetilde{q}_{\gamma}$, solution of $(3.31)$, can be decomposed as $\widetilde{q}_{\gamma}=\widetilde{q}_{\gamma}^{1}+\widetilde{q}_{\gamma}^{2}$, where $\widetilde{q}_{\gamma}^{1}$ is solution to

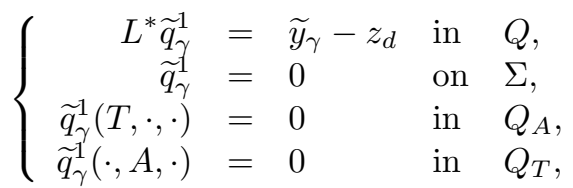

and $\widetilde{q}_{\gamma}^{2}$ is solution to

$$
\left\{\begin{array}{rlrl}
L^{*} \widetilde{q}_{\gamma}^{2} & =\frac{1}{\sqrt{\gamma}} p_{\gamma}+\frac{1}{\gamma} S\left(a ; \widetilde{u}_{\gamma}\right) \xi\left(\widetilde{u}_{\gamma}\right)(t, 0, x) & & \text { in } Q, \\
\widetilde{q}_{\gamma}^{2} & =0 & & \text { on } \Sigma, \\
\widetilde{q}_{\gamma}^{2}(T, \cdot, \cdot) & =0 & \text { in } Q_{A}, \\
\widetilde{q}_{\gamma}^{2}(\cdot, A, \cdot) & =0 & \text { in } Q_{T} .
\end{array}\right.
$$

Proceeding as for $\bar{y}$ in pages $3-4$, while using (3.49), we obtain

$$
\left\|\widetilde{q}_{\gamma}^{1}\right\|_{L^{2}\left(U ; H_{0}^{1}(\Omega)\right)} \leq C\left(T,\|\widetilde{u}\|_{L^{2}\left(Q_{\omega}\right)},\left\|y^{0}\right\|_{L^{2}\left(Q_{A}\right)},\|f\|_{L^{2}(Q)},\left\|z_{d}\right\|_{L^{2}(Q)}\right) .
$$

Combining (3.33) and (3.37), we obtain

$$
\begin{aligned}
0 & =\int_{Q} \bar{y}(w)\left(\widetilde{y}_{\gamma}-z_{d}\right) d t d a d x \\
& +\int_{Q_{\omega}} N \widetilde{u}_{\gamma} w d t d a d x+\int_{Q_{\omega}}\left(\widetilde{u}_{\gamma}-\tilde{u}\right) w d t d a d x \\
& +\int_{Q} \bar{y}(w)\left(\frac{1}{\sqrt{\gamma}} p_{\gamma}+\frac{1}{\gamma} \widetilde{\xi}_{\gamma}(0) S\left(a ; \widetilde{u}_{\gamma}\right)\right) d t d a d x, \forall w \in L^{2}\left(Q_{\omega}\right) .
\end{aligned}
$$

Set

$$
\mathcal{E}=\left\{\bar{y}(w), \quad w \in L^{2}\left(Q_{\omega}\right)\right\} .
$$

Then $\mathcal{E} \subset L^{2}(Q)$. We define on $\mathcal{E} \times \mathcal{E}$ the inner product:

$$
\langle\bar{y}(v), \bar{y}(w)\rangle_{\mathcal{E}}=\int_{Q_{\omega}} v w d t d a d x+\int_{Q} \bar{y}(v) \bar{y}(w) d t d a d x, \forall \bar{y}(v), \bar{y}(w) \in \mathcal{E} .
$$

Then $\mathcal{E}$ endowed with the norm

$$
\|\bar{y}(w)\|_{\mathcal{E}}^{2}=\|w\|_{L^{2}\left(Q_{\omega}\right)}^{2}+\|\bar{y}(w)\|_{L^{2}(Q)}^{2}, \forall \bar{y}(w) \in \mathcal{E}
$$

is a Hilbert space. We set

$$
T_{\gamma}\left(\widetilde{u}_{\gamma}\right)=\frac{1}{\sqrt{\gamma}} p_{\gamma}+\frac{1}{\gamma} \widetilde{\xi}_{\gamma}(0) S\left(a ; \widetilde{u}_{\gamma}\right) .
$$

Then, in view of (3.53), we have for any $w \in L^{2}\left(Q_{\omega}\right)$,

$$
\begin{aligned}
\int_{Q} T_{\gamma}\left(\widetilde{u}_{\gamma}\right) \bar{y}(w) d t d a d x & =-\int_{Q} \bar{y}(w)\left(\widetilde{y}_{\gamma}-z_{d}\right) d t d a d x \\
& -\int_{Q_{\omega}} N \widetilde{u}_{\gamma} w d t d a d x-\int_{Q_{\omega}}\left(\widetilde{u}_{\gamma}-\tilde{u}\right) w d t d a d x .
\end{aligned}
$$


Using the Cauchy Schwarz inequality, we have

$$
\begin{aligned}
& \left|-\int_{Q} \bar{y}(w)\left(\widetilde{y}_{\gamma}-z_{d}\right) d t d a d x-\int_{Q_{\omega}}\left((N+1) \widetilde{u}_{\gamma}-\widetilde{u}\right) w d t d a d x\right| \leq \\
& \left\|\widetilde{y}_{\gamma}-z_{d}\right\|_{L^{2}(Q)}\|\bar{y}(w)\|_{L^{2}(Q)}+(N+1)\left\|\widetilde{u}_{\gamma}\right\|_{L^{2}\left(Q_{\omega}\right)}\|w\|_{L^{2}\left(Q_{\omega}\right)}+ \\
& \|\widetilde{u}\|_{L^{2}\left(Q_{\omega}\right)}\|w\|_{L^{2}\left(Q_{\omega}\right)} .
\end{aligned}
$$

Therefore, using (3.49) and (3.39),

$$
\begin{aligned}
& \left|-\int_{Q} \bar{y}(w)\left(\widetilde{y}_{\gamma}-z_{d}\right) d t d a d x-\int_{Q_{\omega}}\left((N+1) \widetilde{u}_{\gamma}-\widetilde{u}\right) w d t d a d x\right| \leq \\
& \left(\left\|\widetilde{y}_{\gamma}-z_{d}\right\|_{L^{2}(Q)}^{2}+\left[(N+1)\left\|\widetilde{u}_{\gamma}\right\|_{L^{2}\left(Q_{\omega}\right)}+\|\widetilde{u}\|_{L^{2}\left(Q_{\omega}\right)}\right]^{2}\right)^{1 / 2}\|\bar{y}(w)\|_{\mathcal{E}} \leq \\
& C\|\bar{y}(w)\|_{\mathcal{E}},
\end{aligned}
$$

where $C=C\left(N, T,\|\widetilde{u}\|_{L^{2}\left(Q_{\omega}\right)},\left\|y^{0}\right\|_{L^{2}\left(Q_{A}\right)},\|f\|_{L^{2}(Q)},\left\|z_{d}\right\|_{L^{2}(Q)}\right)>0$. It then follows from (3.57)

Consequently,

$$
\left|\int_{Q} T_{\gamma}\left(\widetilde{u}_{\gamma}\right) \bar{y}(w) d t d a d x\right| \leq C\|\bar{y}(w)\|_{\mathcal{E}}
$$

$$
\left\|T_{\gamma}\left(\widetilde{u}_{\gamma}\right)\right\|_{\mathcal{E}^{\prime}}=\left\|\frac{1}{\sqrt{\gamma}} p_{\gamma}+\frac{1}{\gamma} \widetilde{\xi}_{\gamma}(0) S\left(a ; \widetilde{u}_{\gamma}\right)\right\|_{\mathcal{E}^{\prime}} \leq C
$$

In particular,

$$
\left\|\frac{1}{\sqrt{\gamma}} p_{\gamma}+\frac{1}{\gamma} \widetilde{\xi}_{\gamma}(0) S\left(a ; \widetilde{u}_{\gamma}\right)\right\|_{L^{2}(Q)} \leq C,
$$

where $C=C\left(N, T,\|\widetilde{u}\|_{L^{2}\left(Q_{\omega}\right)},\left\|y^{0}\right\|_{L^{2}\left(Q_{A}\right)},\|f\|_{L^{2}(Q)},\left\|z_{d}\right\|_{L^{2}(Q)}\right)>0$.

Now, proceeding as for $\bar{y}$ in pages $3-4$, while using (3.58), we obtain that

$$
\left\|\widetilde{q}_{\gamma}^{2}\right\|_{L^{2}\left(U ; H_{0}^{1}(\Omega)\right)} \leq C\left(N, T,\|\widetilde{u}\|_{L^{2}\left(Q_{\omega}\right)},\left\|y^{0}\right\|_{L^{2}\left(Q_{A}\right)},\|f\|_{L^{2}(Q)},\left\|z_{d}\right\|_{L^{2}(Q)}\right) .
$$

Finally from (3.52) and (3.59) we deduce (3.47).

\subsection{Characterization of the No-regret control.}

Proposition 3.12. The adapted Low-regret optimal control $\widetilde{u}_{\gamma}$ converges in $L^{2}\left(Q_{\omega}\right)$ to the No-regret control $\widetilde{u} \in \mathcal{M}$.

Proof. In view of (3.39)-(3.44), there exists a subsequence of $\left(\widetilde{u}_{\gamma}, \widetilde{y}_{\gamma}, \widetilde{\xi}_{\gamma}, S\left(., \widetilde{u}_{\gamma}\right)\right)$ still denoted by $\left(\widetilde{u}_{\gamma}, \widetilde{y}_{\gamma}, \widetilde{\xi}_{\gamma}, S\left(., \widetilde{u}_{\gamma}\right)\right)$ and $\hat{u} \in L^{2}\left(Q_{\omega}\right), \widetilde{y} \in L^{2}\left(U, H_{0}^{1}(\Omega)\right), \widetilde{\xi} \in L^{2}\left(U, H_{0}^{1}(\Omega)\right)$, $\alpha \in L^{2}(0, A), \tau \in L^{2}(Q T)$ such that

$$
\begin{aligned}
& \widetilde{u}_{\gamma} \rightarrow \hat{u} \text { weakly in } L^{2}\left(Q_{\omega}\right), \\
& \left.\frac{1}{\sqrt{\gamma}} S\left(., \widetilde{u}_{\gamma}\right)\right) \rightarrow \alpha \text { weakly in } L^{2}(0, A) \text {, } \\
& \left.S\left(., \widetilde{u}_{\gamma}\right)\right) \rightarrow 0 \text { strongly in } L^{2}(0, A), \\
& \widetilde{y}_{\gamma} \rightarrow \widetilde{y} \text { weakly in } L^{2}\left(U ; H_{0}^{1}(\Omega)\right) \text {, } \\
& \widetilde{\xi}_{\gamma} \rightarrow \widetilde{\xi} \text { weakly in } L^{2}\left(U ; H_{0}^{1}(\Omega)\right), \\
& \widetilde{\xi}_{\gamma}(., 0, .) \rightarrow \tau \text { weakly in } L^{2}\left(Q_{T}\right) .
\end{aligned}
$$


If we multiply the first equation (3.28) by $\phi \in \mathcal{D}(Q)$ and the first equation in (3.29) by $\psi \in \mathcal{D}(Q)$ and integrate by parts over $Q$, we have

$$
\int_{Q} \widetilde{y}_{\gamma} L^{*} \phi d t d a d x=\int_{Q}\left(f+\widetilde{u}_{\gamma} \chi_{\omega}\right) \phi d t d a d x
$$

and

$$
\int_{Q} \widetilde{\xi}_{\gamma} L \psi d t d a d x=\int_{Q}\left(\widetilde{y}_{\gamma}-z_{d}\right) \psi d t d a d x
$$

Passing in these two latter identities to the limit, while using (3.60), (3.63) and (3.64), we obtain

$$
\int_{Q} \widetilde{y} L^{*} \phi d t d a d x=\int_{Q}\left(f+\hat{u} \chi_{\omega}\right) \phi d t d a d x
$$

and

$$
\int_{Q} \widetilde{\xi} L \psi d t d a d x=\int_{Q}\left(\widetilde{y}-z_{d}\right) \psi d t d a d x
$$

which after an integration by parts over $Q$ give

$$
\int_{Q} L \widetilde{y} \phi d t d a d x=\int_{Q}\left(f+\hat{u} \chi_{\omega}\right) \phi d t d a d x, \quad \forall \phi \in \mathcal{D}(Q)
$$

and

$$
\int_{Q} L^{*} \widetilde{\xi} \psi d t d a d x=\int_{Q}\left(\widetilde{y}-z_{d}\right) \psi d t d a d x, \quad \forall \psi \in \mathcal{D}(Q)
$$

respectively. Hence, we can deduce

$$
L \widetilde{y}=f+\hat{u} \chi_{\omega} \text { in } Q
$$

and

$$
L^{*} \widetilde{\xi}=\widetilde{y}-z_{d} \text { in } Q .
$$

Note that $\widetilde{y}, \widetilde{\xi} \in L^{2}\left(U, H_{0}^{1}(\Omega)\right)$. This implies that $\left.\widetilde{y}(t, a)\right|_{\Gamma}$ and $\left.\widetilde{\xi}(t, a)\right|_{\Gamma}$ exist and belong to $L^{2}(\Gamma)$ for almost every $(t, a) \in U$. On the other hand from (3.66), (3.67) and the expression of the operator $L$ and $L^{*}$ given by (2.1), we have $\widetilde{y}, \widetilde{\xi} \in W(T, A)$. It follows from Remark 1.1 that $(\widetilde{y}(0, .),. \widetilde{\xi}(T, .,)$.$) exists and belongs \left(L^{2}\left(Q_{A}\right)\right)^{2}$ and $(\widetilde{y}(., 0,),. \widetilde{\xi}(., A,),. \widetilde{\xi}(., 0,)$.$) exists and belongs \left(L^{2}\left(Q_{T}\right)\right)^{2}$.

Now, if we multiply the first equation (3.28) by $\phi \in \mathcal{C}^{\infty}(\bar{Q})$ such that $\phi=0$ on $\Sigma, \phi(\cdot, A, \cdot)=0$ in $Q_{T}$ and $\phi(T, \cdot, \cdot)=0$ in $Q_{A}$ and the first equation in (3.29) by $\psi \in \mathcal{C}^{\infty}(\bar{Q})$ such that $\psi=0$ on $\Sigma$ and $\psi(0, \cdot, \cdot)=0$ in $Q_{A}$ and integrate by parts over $Q$, we respectively have that

$$
-\int_{Q_{A}} y^{0} \phi(0, a, x) d a d x+\int_{Q} \widetilde{y}_{\gamma} L^{*} \phi d a d x=\int_{Q}\left(f+\widetilde{u}_{\gamma} \chi_{\omega}\right) \phi d t d a d x
$$

and

$$
\int_{Q_{A}} \widetilde{\xi}_{\gamma}(t, 0, x) \psi(0, a, x) d t d x+\int_{Q} \widetilde{\xi}_{\gamma} L \psi d a d x=\int_{Q}\left(\widetilde{y}_{\gamma}-z_{d}\right) \psi d t d a d x .
$$


Passing these two latter identities to the limit while using (3.60), (3.63), (3.64) and (3.65), we obtain

$$
\begin{aligned}
& -\int_{Q_{A}} y^{0} \phi(0, a, x) d a d x+\int_{Q} \widetilde{y} L^{*} \phi d t d a d x=\int_{Q}\left(f+\hat{u} \chi_{\omega}\right) \phi d t d a d x, \\
& \forall \phi \in \mathcal{C}^{\infty}(\bar{Q}) \text { such that }\left.\phi\right|_{\Gamma}=0,\left.\phi(\cdot, A, \cdot)\right|_{Q_{T}}=0,\left.\phi(T, \cdot, \cdot)\right|_{Q_{A}}=0,
\end{aligned}
$$

and

$$
\begin{aligned}
& \int_{Q_{A}} \tau \psi(0, a, x) d t d x+\int_{Q} \widetilde{\xi} L \psi d t d a d x=\int_{Q}\left(\widetilde{y}-z_{d}\right) \psi d t d a d x, \\
& \forall \psi \in \mathcal{C}^{\infty}(\bar{Q}) \text { such that }\left.\psi\right|_{\Gamma}=0,\left.\psi(0, \cdot, \cdot)\right|_{Q_{A}}=0,
\end{aligned}
$$

respectively, which after an integration by parts over $Q$ give

$$
\begin{aligned}
& \int_{Q}\left(f+\hat{u} \chi_{\omega}\right) \phi d t d a d x=-\int_{Q_{A}}\left(y^{0}-\widetilde{y}(0, a, x)\right) \phi(0, a, x) d a d x+ \\
& \int_{Q_{T}} \widetilde{y}(t, 0, x) \phi(t, 0, x) d t d x-\int_{\Sigma} \widetilde{y} \frac{\partial \phi}{\partial \nu} d t d a d x+\int_{Q} L \widetilde{y} \phi d t d a d x, \\
& \forall \phi \in \mathcal{C}^{\infty}(\bar{Q}) \text { such that }\left.\phi\right|_{\Gamma}=0,\left.\phi(\cdot, A, \cdot)\right|_{Q_{T}}=0,\left.\phi(T, \cdot, \cdot)\right|_{Q_{A}}=0,
\end{aligned}
$$

and

$$
\begin{aligned}
& \int_{Q}\left(\widetilde{y}-z_{d}\right) \psi d t d a d x=\int_{Q_{A}}(\tau-\widetilde{\xi}(t, 0, x)) \psi(0, a, x) d t d x+ \\
& \int_{Q} L^{*} \widetilde{\xi} \psi d t d a d x+\int_{Q_{A}} \widetilde{\xi}(T, a, x) \psi(T, a, x) d a d x+ \\
& \int_{Q_{T}} \widetilde{\xi}(t, A, x) \psi(t, A, x) d t d x+\int_{\Sigma} \widetilde{\xi} \frac{\partial \psi}{\partial \nu} d t d a d x, \\
& \forall \psi \in \mathcal{C}^{\infty}(\bar{Q}) \text { such that }\left.\psi\right|_{\Gamma}=0,\left.\psi(0, \cdot, \cdot)\right|_{Q_{A}}=0 .
\end{aligned}
$$

Using (3.66) and (3.67), we deduce from these latter identities that,

$$
\begin{aligned}
& 0=-\int_{Q_{A}}\left(y^{0}-\widetilde{y}(0, a, x)\right) \phi(0, a, x) d a d x+ \\
& \int_{Q_{T}} \widetilde{y}(t, 0, x) \phi(t, 0, x) d t d x-\int_{\Sigma} \widetilde{y} \frac{\partial \phi}{\partial \nu} d t d a d x, \\
& \forall \phi \in \mathcal{C}^{\infty}(\bar{Q}) \text { such that }\left.\phi\right|_{\Gamma}=0,\left.\phi(\cdot, A, \cdot)\right|_{Q_{T}}=0,\left.\phi(T, \cdot, \cdot)\right|_{Q_{A}}=0,
\end{aligned}
$$

377 and

$$
\begin{aligned}
& 0=\int_{Q_{A}}(\tau-\widetilde{\xi}(t, 0, x)) \psi(0, a, x) d t d x+ \\
& \int_{Q_{A}} \widetilde{\xi}(T, a, x) \psi(T, a, x) d a d x+ \\
& \int_{Q_{T}} \widetilde{\xi}(t, A, x) \psi(t, A, x) d t d x+\int_{\Sigma} \widetilde{\xi} \frac{\partial \psi}{\partial \nu} d t d a d x, \\
& \forall \psi \in \mathcal{C}^{\infty}(\bar{Q}) \text { such that }\left.\psi\right|_{\Gamma}=0,\left.\psi(0, \cdot \cdot \cdot)\right|_{Q_{A}}=0 .
\end{aligned}
$$

If we successively take in (3.68) and (3.69),

$$
\begin{aligned}
& \left.\phi(\cdot, 0, \cdot)\right|_{Q_{T}}=0 \text { and }\left.\frac{\partial \phi}{\partial \nu}\right|_{\Gamma}=0 \\
& \left.\psi(\cdot, 0, \cdot)\right|_{Q_{T}}=\left.\psi(\cdot, A, \cdot)\right|_{Q_{T}}=0 \text { and }\left.\frac{\partial \psi}{\partial \nu}\right|_{\Gamma}=0,
\end{aligned}
$$


we successively obtain

$$
\begin{aligned}
& \left.\frac{\partial \phi}{\partial \nu}\right|_{\Gamma}=0, \\
& \left.\psi(\cdot, A, \cdot)\right|_{Q_{T}}=0 \text { and }\left.\frac{\partial \psi}{\partial \nu}\right|_{\Gamma}=0,
\end{aligned}
$$

then in (3.69),

$$
\left.\frac{\partial \psi}{\partial \nu}\right|_{\Gamma}=0
$$

then

$$
\begin{gathered}
\widetilde{y}(0, \cdot, \cdot)=y^{0} \text { in } Q_{A}, \\
\widetilde{\xi}(T, \cdot, \cdot)=0 \text { in } Q_{A}, \\
\widetilde{y}(\cdot, 0, \cdot)=0 \text { in } Q_{T}, \\
\widetilde{\xi}(\cdot, A, \cdot)=0 \text { in } Q_{T},
\end{gathered}
$$

and finally,

$$
\begin{aligned}
\widetilde{y} & =0 \text { on } \Sigma, \\
\widetilde{\xi}(\cdot, 0, \cdot) & =\tau \text { in } Q_{T},
\end{aligned}
$$

Now, using (3.60),(3.63), (3.65), (3.75) and (3.41), we have from Lemma 3.5 that

$$
S\left(., u^{\gamma}\right) \rightarrow S(., \hat{u}) \text { weakly in } \mathcal{D}^{\prime}(0, A) .
$$

Hence, using (3.62) and the uniqueness of the limit that

$$
S\left(., \widetilde{u}_{\gamma}\right) \rightarrow S(., \hat{u})=0 \text { strongly in } L^{2}(0, A) .
$$

Consequently,

$$
\int_{0}^{A} S\left(a ; \widetilde{u}_{\gamma}\right) g(a) d a \rightarrow \int_{0}^{A} S\left(a ; \hat{u}_{\gamma}\right) g(a) d a=0 .
$$

Thus $\hat{u} \in \mathcal{M}$ and we also have $\|S(. ; \hat{u})\|_{L^{2}(0, A)}=0$. Since $\widetilde{u}$ is a No-regret control and $\widehat{u} \in \mathcal{M}$, it follows from (3.21) that

$$
J(\widetilde{u}, 0)-J(0,0) \leq J(\widehat{u}, 0)-J(0,0),
$$

Observing that $\widetilde{u}_{\gamma}$ solves the problem $\inf _{v \in L^{2}\left(Q_{\omega}\right)} \widetilde{\mathcal{J}}^{\gamma}(v)$, we have

$$
\widetilde{\mathcal{J}}^{\gamma}\left(\widetilde{u}_{\gamma}\right) \leq \widetilde{\mathcal{J}}^{\gamma}(\widetilde{u})=J(\widetilde{u}, 0)-J(0,0),
$$

which, in view of the definition of $\widetilde{\mathcal{J}}^{\gamma}$ given by (3.26), implies that

$$
J\left(\widetilde{u}_{\gamma}, 0\right)-J(0,0)+\left\|\widetilde{u}_{\gamma}-\widetilde{u}\right\|_{L^{2}\left(Q_{\omega}\right)}^{2} \leq \widetilde{\mathcal{J}}^{\gamma}\left(\widetilde{u}_{\gamma}\right) \leq \widetilde{\mathcal{J}}^{\gamma}(\widetilde{u})=J(\widetilde{u}, 0)-J(0,0) .
$$

Using the convexity and lower semi-continuity of $J$ on $L^{2}\left(Q_{\omega}\right),(3.60)$ and (3.63), we obtain

$$
J(\hat{u}, 0)-J(0,0)+\|\hat{u}-\widetilde{u}\|_{L^{2}\left(Q_{\omega}\right)}^{2} \leq \liminf _{\gamma \rightarrow 0} \widetilde{\mathcal{J}}^{\gamma}\left(\widetilde{u}_{\gamma}\right) \leq J(\widetilde{u}, 0)-J(0,0),
$$


which combining with (3.77) gives

$$
\|\hat{u}-\widetilde{u}\|_{L^{2}\left(Q_{\omega}\right)}^{2} \leq 0 .
$$

Hence,

$$
\hat{u}=\widetilde{u} \text { in } Q_{\omega} .
$$

Thus the adapted Low-regret controls converge in $L^{2}\left(Q_{\omega}\right)$ to the No-regret control. Moreover from (3.80), (3.66), (3.70), (3.72) and (3.74), it follows that $\widetilde{y}=y(\widetilde{u}, 0) \in$ $L^{2}\left(U ; H_{0}^{1}(\Omega)\right)$ unique solution of

$$
\left\{\begin{aligned}
L \widetilde{y} & =f+\widetilde{u} \chi_{Q_{\omega}} & & \text { in } \quad Q, \\
\widetilde{y} & =0 & & \text { on } \Sigma, \\
\widetilde{y}(0, \cdot, \cdot) & =y^{0} & & \text { in } \\
\widetilde{y}(\cdot, 0, \cdot) & =0 & & \text { in } \quad Q_{T} .
\end{aligned}\right.
$$

Similarly, from (3.67), (3.71),(3.73) and (3.76), we infer that $\widetilde{\xi}=\xi(\widetilde{u})$ is the unique solution of

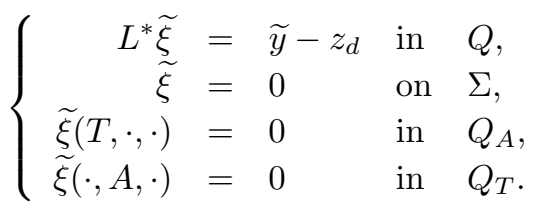

Proposition 3.13. The No-regret control $\widetilde{u} \in \mathcal{M}$ is characterized by the functions $\widetilde{u}, \widetilde{y}, \widetilde{\xi}, \widetilde{p}$ and $\widetilde{q}$ which are unique solutions of the optimality system:

$$
\left\{\begin{aligned}
L \widetilde{y} & =f+\widetilde{u} \chi_{Q_{\omega}} & & \text { in } \quad Q, \\
\widetilde{y} & =0 & & \text { on } \Sigma, \\
\widetilde{y}(0, \cdot, \cdot) & =y^{0} & & \text { in } Q_{A}, \\
\widetilde{y}(\cdot, 0, \cdot) & =0 & & \text { in } Q_{T},
\end{aligned}\right.
$$

$$
\left\{\begin{array}{rlrl}
L^{*} \widetilde{\xi} & =\widetilde{y}-z_{d} & \text { in } \quad & Q, \\
\widetilde{\xi} & =0 & \text { on } \Sigma, \\
\widetilde{\xi}(T, \cdot, \cdot) & =0 & \text { in } \quad Q_{A}, \\
\widetilde{\xi}(\cdot, A, \cdot) & =0 & \text { in } \quad Q_{T},
\end{array}\right.
$$

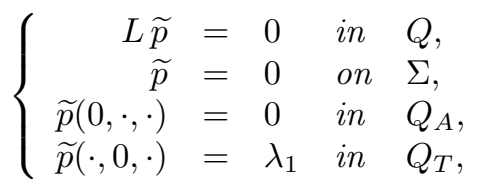

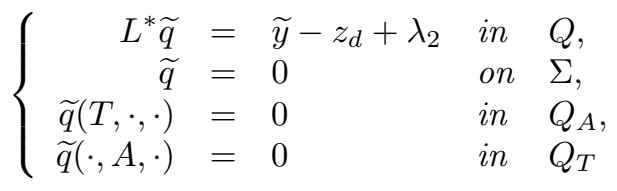


and

$$
N \widetilde{u}+\widetilde{q}=0,
$$

where

$$
\begin{aligned}
& \lambda_{1}=\lim _{\gamma \rightarrow 0} \frac{1}{\sqrt{\gamma}} \int_{0}^{A} y_{\gamma}\left(t, a, x, \widetilde{u}_{\gamma}\right) S\left(a ; \widetilde{u}_{\gamma}\right) d a, \\
& \lambda_{2}=\lim _{\gamma \rightarrow 0}\left(\frac{1}{\sqrt{\gamma}} p_{\gamma}+\frac{1}{\gamma} \widetilde{\xi}_{\gamma}(0) S\left(a ; \widetilde{u}_{\gamma}\right)\right) .
\end{aligned}
$$

Proof. We have already proved (3.83) and (3.84) (see Page 23).

From (3.45), (3.58),(3.46) and (3.47), we have there exist $\lambda_{1} \in L^{2}\left(Q_{T}\right), \lambda_{2} \in$ $L^{2}(Q), \widetilde{p} \in L^{2}\left(U ; H_{0}^{1}(\Omega)\right)$ and $\widetilde{q} \in L^{2}\left(U ; H_{0}^{1}(\Omega)\right)$ such that

$$
\begin{aligned}
\widetilde{p}_{\gamma}(., 0, .) & \rightarrow \lambda_{1} \text { in } L^{2}\left(Q_{T}\right), \\
\frac{1}{\sqrt{\gamma}} p_{\gamma}+\frac{1}{\gamma} \widetilde{\xi}_{\gamma}(0) S\left(a ; \widetilde{u}_{\gamma}\right) & \rightarrow \lambda_{2} \text { in } L^{2}(Q), \\
\widetilde{p}_{\gamma} & \rightarrow \widetilde{p} \text { in } L^{2}\left(U ; H_{0}^{1}(\Omega)\right), \\
\widetilde{q}_{\gamma} & \rightarrow \widetilde{q} \text { in } L^{2}\left(U ; H_{0}^{1}(\Omega)\right) .
\end{aligned}
$$

Then, proceeding as for $\widetilde{y}_{\gamma}$ and $\widetilde{\xi}_{\gamma}$ in Pages 19-22 while using (3.89)-(3.91), we prove (3.85) and (3.86). To obtain (3.87), we pass to the limit in (3.38) while using (3.60), (3.80) and (3.91).

4. Conclusions. We used the notion of No-regret and Low-regret to control a model describing the dynamics of population with age dependence and spatial structure with missing birth rate. In contrast to some works on the topic which need the control to act on the whole domain to obtain the convergence of the optimality system that characterizes the Low-regret control towards the singular optimality characterizing the No-regret control, our control acts on a part of the domain. We then introduce an appropriate Hilbert space and apply the Aubin-Lions Lemma to an appropriate auxiliary problem to obtain the convergence of an adapted Low-regret control towards a No-regret control that we characterize.

Acknowledgments. The third author was supported by the Alexander von Humboldt foundation, under the programme financed by the BMBF entitled "German research Chairs". The first author is grateful for the facilities provided by the German research Chairs. The second author was supported by the DFG-TRR 154 "Modellierung Simulation und Optimierung am Beispiel von Gasnetzwerken" (TPA05),

[1] J. -L. Lions, Contrôle à moindre regrets des systèmes distribués, C. R. Acad. Sci. Paris Sr. I, 315(1992), pp. 1253-1257.

[2] W. Rundel, Determining the birth function fo an age structured population, Mathematical Population Studies, 1989, Vol 1(4), pp. 377-395.

[3] B. Ainseba And M. Langlais, On a population dynamics control problem with age dependence and spatial structure, J. Math. Anal. Appl., 248(2000), pp. 455-474.

[4] B.AinsebA AND S.Anita, Local exact controllability of the age-dependent population dynamics with diffusion,Abstract Appl.Anal, 6(2001), pp. 357-368.

[5] A. Ouedraogo And O. Traoré, Optimal control for a nonlinear population dynamics problem,Portugaliae Mathematica, 62(2005), pp.217-229.

[6] M. LAnglais, A nonlinear problem in age-dependent population diffusion, SIAM J. Math. Anal., 16(1985), pp. 510-529. 
[7] G.F. WebB, Theory of Age Nonlinear Population Dynamics, Marcel Dekker, New York, 1985.

[8] M.G. Garroni And M. Langlais, Age-dependent population diffusion with external Constraint, J. Math. Biol., 14(1982), pp. 77-94.

[9] A. Ouédraogo, O. Traoré, Sur un problème non linéaire de dynamique des populations, IMHOTEP, 4(2003), pp. 15-23.

[10] M. Langlais, Solutions fortes pour une classe de problèmes aux limites dégénérés, Comm. in Partial Differential Equations 4 (8)(1979), 869-897.

[11] J. -L. LiOns AND J. -I DiAZ, Environment, economics and their mathematical models, Masson, Paris, 1994.

[12] O. Nakoulima, A. Omrane and J. Velin, On the pareto control and No-regret control for distributed systems with incomplete data, SIAM J. Control Optim., 42(2003), pp. 11671184.

[13] B. JacoB AND A. OmRane, Optimal control for age-structured population dynamics of incomplete data, J. Math. Anal. Appl., 370(2010), pp. 42-48.

[14] O. Nakoulima, A. Omrane and J. Velin, No-regret control for nonlinear distributed systems with incomplete data, J. Math. Pures Appl., 81(2002), pp. 1161-1189.

[15] J. VELIN, No-regret distributed control of system governed by quasilinear elliptic equations with incomplete data: the degenerate case, J. Math. Pures Appl., 83(2004), pp. 503-539.

[16] D. AzÉ, Éléments d'analyse convexe et variationnelle, ellipses , Paris, 1997.

[17] J. -L. Lions, Least regret control, virtual control and decomposition methods, Mathematical Modelling and Numerical Analysis. M2AN, 34(2000), 409418. 